\title{
HARMONIC FUNCTIONS OF RANDOM WALKS IN A SEMIGROUP VIA LADDER HEIGHTS.
}

\author{
IRINA IGNATIOUK-ROBERT
}

\begin{abstract}
We investigate harmonic functions and the convergence of the sequence of ratios $\left(\mathbb{P}_{x}\left(\tau_{\vartheta}>n\right) / \mathbb{P}_{e}\left(\tau_{\vartheta}>n\right)\right)$ for a random walk on a countable group killed up on the time $\tau_{\vartheta}$ of the first exit from some semi-group with an identity element $e$ Several results of classical renewal theory for one dimensional random walk killed at the first exit from the positive half-line are extended to a multi-dimensional setting. For this purpose, an analogue of the ladder height process and the corresponding renewal function $V$ are introduced. The results are applied to multidimensional random walks $(X(t))$ killed upon the times of first exit from a convex cone. Our approach combines large deviation estimates and an extension of Choquet-Deny theory.
\end{abstract}

\section{INTRODUCTION}

An explicit description of all harmonic functions for a transient countable Markov chain is in general a non trivial problem. Markov chains associated to homogeneous random walks are one of the few examples when such a complete description can be obtained. This is done via the classical method of Choquet-Deny theory, see for example Sawyer 1].

For a non homogeneous transient Markov chain with a transition kernel $P$, the method of Choquet-Deny theory does not work. To find all harmonic functions one has either to solve the equation $P h=h$ in a straightforward way via analytical methods, or to investigate the Martin boundary of the process, by identifying all possible limits of the Martin kernel. The classical references for the second approach are Doob [2, Hunt 3 ] and Dynkin 4. Both approaches are non-trivial and there are also few examples when all harmonic functions can be found.

A simple but important example of a non-homogeneous Markov chain is a random walk on some group $(\mathcal{E}, \star)$ killed up on the time $\tau_{\vartheta}$ of the first exit from some semi-group $E \subset \mathcal{E}$. The harmonic functions of such a random walk are related to a random walk conditioned on the event $\left\{\tau_{\vartheta}>n\right\}$ when $n \rightarrow \infty$, i.e. to stay in $E$ : if the sequence of ratios $\left(\mathbb{P}_{x}\left(\tau_{\vartheta}>n\right) / \mathbb{P}_{e}\left(\tau_{\vartheta}>n\right)\right)$ converges to some harmonic function $(h(x))$, the corresponding conditioned random walk is the $h$-transform of the original killed random walk. Random walks conditioned to stay in cones have been investigated in a large number of references, see for instance Bertoin and Doney [5] and Denisov and Wachtel [6] and the references therein.

For one-dimensional random walks with a non-negative drift, killed up on the time of the first exit from a half line, the harmonic functions and the corresponding random walk conditioned to stay in the half line can be represented in terms of the renewal function based on the ladder heights by using the Wiener-Hopf factorization. The method of the Wiener-Hopf factorization is a quite powerful technique. It does not require any moment conditions in the one-dimensional case, see for instance Spitzer 8], Feller 9] and Bertoin and Doney [5]. For multidimensional random walks, a similar approach

Date: Received: date / Accepted: date.

1991 Mathematics Subject Classification. 60J45, 31C05, 60J10.

Key words and phrases. Harmonic function, random walk, exit time, renewal function. 
seems to be unlikely because there is, up to now, no equivalent of a convenient WienerHopf factorization. There are nevertheless some results in this domain, Mogulskii and Pecherskii in [10] and Greenwood and Shaked 11, 12] where some factorization relations are established to get the asymptotic behavior of some first passage times. It is not clear however how these factorization relations can be used to investigate the harmonic functions and the corresponding random walk conditioned to stay in a cone.

We review briefly several approaches used to identify the set of harmonic functions and to investigate the limit of the sequence $\left(\mathbb{P}_{x}\left(\tau_{\vartheta}>n\right) / \mathbb{P}_{e}\left(\tau_{\vartheta}>n\right)\right)$ for multidimensional random walks.

To identify the Martin boundary for integer-valued two-dimensional random walks when the size of the jumps of each coordinate is at most 1, complex analysis methods on elliptic curves has been proposed by Kurkova and Malyshev [13, Kurkova and Raschel 14 . and Raschel [15. This approach does not seem to have an extension in higher dimensions or for random walks with unbounded jumps.

A large deviations approach has been proposed in Ignatiouk and Loree [16 to identify the Martin boundary of two-dimensional, non centered, random walks killed up on the first exit from the positive quadrant $\mathbb{N} \times \mathbb{N}$. For centered random walks, such a method does not seem to work. Moreover, to apply such a method for a non centered random walk in $\mathbb{Z}_{+}^{d}$, one has to identify the harmonic functions for centered random walks in $\mathbb{Z}_{+}^{k}$ for any $k<d$, see Ignatiouk [17.

Recently, Bouaziz et al. [18] proved the uniqueness of the positive harmonic function, up to a multiplicative constant, for a centered random walk killed up on the time of the first exit from $\mathbb{Z}_{+}^{d}$. The result has been obtained under the following conditions: the jumps of the random walk must be bounded and their distribution has to satisfy some ellipticity condition. A large number of related results were obtained for random walks in Weyl chambers, see for instance Gessel and Zeilberger [19], Grabiner and Magyar [20], Koenig and Schmid 21] and Raschel [22, 23, and the references therein. These results concern some specific random walks, when the steps of the process cannot jump over the boundary of the chamber and when the set of all possible steps is invariant with respect to some reflections.

For centered random walks killed up on the time of the first exit from some general cone, under some moment condition on the jumps, a harmonic function has been constructed and the asymptotic of the tail distribution of the exit time has been obtained by Denisov and Wachtel [6], and in a recent paper of Raschel and Tarrago [7] the asymptotic behavior of the Green function was obtained and a uniqueness (up to a multiplicative constant) of a positive harmonic function was proved. For non-centered random walks satisfying a Cramer condition the results of Denisov and Wachtel [6] have been extended by Duraj [24. These results use the diffusion approximation of random walks and seem to be impossible to extend for random walks with heavy tailed jumps.

The purpose of the present paper is to extend the notions of the ladder heights and of the corresponding renewal function to a multi-dimensional setting, and to obtain results similar to those in the classical fluctuation theory for one dimensional random walks without any assumption on the tail distributions of the jumps. For this we combine the large deviation estimates with a modified method of Choquet-Deny theory.

We consider a substochastic transient random walk on a countable semi-group $(E, \star)$ with identity element $e$. The transition probabilities of our random walk are assumed to satisfy the inequalities $p(x \star u, y \star u) \geq p(x, y)$ for all $x, y, u \in E$. A homogeneous random walk on $\mathbb{Z}^{d}$ killed up on the time of the first exit from some convex cone with a vertex at the origin 0 is therefore a particular case.

To define the ladder height process and the corresponding renewal function $V$, an additional absorbing state $\vartheta$ is added so that our random walk $(X(t))$ has a stochastic kernel, $\tau_{\vartheta}$ is defined as the hitting time of $\vartheta$. For a random walk on $\mathbb{Z}^{d}$ killed up on the time of the first exit from the cone, this additional state $\vartheta$ is in fact identified with the points 
of $\mathbb{Z}^{d}$ outside of the cone and $\tau_{\vartheta}$ represents the time of the first exit from the cone. When the transition probabilities of our random walk satisfy the identities $p(x \star u, y \star u)=p(x, y)$ for all $x, y, u \in E$, the ladder height process $\left(H_{n}\right)$ has a simple probabilistic interpretation: for a sequence of stopping times $\left(t_{n}\right)$ with $t_{0}=0$ and $t_{n}$ defined as the first time after $t_{n-1}$ when the random walk $(X(t))$ exits from the set $E \star X\left(t_{n-1}\right)$, in distribution $H_{n}=X\left(t_{n}\right)$ if $t_{n}<+\infty$ and $H_{n}=\vartheta$ otherwise. In the general case, the ladder height process is defined by positive operators represented in terms of the differences $p(x \star u, y \star u)-p(x, y)$.

For a random walk in $\mathbb{Z}$, killed up on the first exit from $\mathbb{Z}_{+}$, i.e. when $(\mathcal{E}, \star)=(\mathbb{Z},+)$, our definition of the ladder height process $\left(H_{n}\right)$ coincides with the classical definition. However, in contrast to the ladder height process for a one dimensional random walk, for a multidimensional random walk, the ladder height process is no longer decreasing. This is the main technical difficulty in our approach.

The renewal function $V$ corresponding to the ladder height process is defined then by $V(x)=\mathbb{E}_{x}\left(\mathcal{T}_{\vartheta}\right)$ where $\mathcal{T}_{\vartheta}$ denotes the first time when the process $\left(H_{n}\right)$ hits the absorbing state $\vartheta$. When the stopping time $\tau_{\vartheta}$ is not integrable we prove that the renewal function $V$ is harmonic for the random walk $(X(t))$, and that the sequence of functions

$$
\mathbb{P}_{x}\left(\tau_{\vartheta}>n\right) / \mathbb{P}_{e}\left(\tau_{\vartheta}>0\right), \quad x \in E .
$$

converges to $V$. When the stopping time $\tau_{\vartheta}$ is integrable, an analogue of the Wald identity

$$
\mathbb{E}_{x}\left(\tau_{\vartheta}\right)=V(x) \mathbb{E}_{e}\left(\tau_{\vartheta}\right), \quad \forall x \in E,
$$

is obtained, and we show that

$$
V(x) \leq \liminf _{n \rightarrow \infty} \mathbb{P}_{x}\left(\tau_{\vartheta}>n\right) / \mathbb{P}_{e}\left(\tau_{\vartheta}>n\right) .
$$

The harmonic functions are investigated in a particular case, when the hitting probabilities of the random walk $(X(t))$ are slowly varying, i.e. when for any $u \in E$ and uniformly on $x \in E$,

$$
\begin{aligned}
& \lim _{n \rightarrow \infty} \frac{1}{n} \log \mathbb{P}_{x}\left(X(t)=x \star u^{\star n}, \text { for some } t \geq 1\right)= \\
& \qquad \lim _{n \rightarrow \infty} \frac{1}{n} \log \mathbb{P}_{x \star u}{ }^{\star n}(X(t)=x, \text { for some } t \geq 1)=0,
\end{aligned}
$$

by using a modified method of Choquet-Deny theory.

In the classical homogeneous setting the main idea of the Choquet-Deny theory is the following: to identify the set of harmonic functions one has to determine the minimal harmonic functions. A harmonic function $h$ is minimal if, for any other harmonic function $\tilde{h}$, the inequality $\tilde{h} \leq h$ implies that $\tilde{h}=c h$ for some constant $c$. If a harmonic function $h$ is minimal for an irreducible homogeneous random walk on a group $(\mathcal{E}, \star)$, then for any $u \in \mathcal{E}$, the function $\tilde{h}(x)=h(x \star u)$ is harmonic and by Harnack's inequality, satisfies the inequality $\delta_{u} \tilde{h} \leq h$ for some constant $\delta_{u}>0$. Hence, for any $x \in E, h(x \star u)=\gamma_{u} h(x)$ for some constant $\gamma_{u}>0$. The last relation gives a characterization of all minimal harmonic functions. For a homogeneous random walk in $\mathbb{Z}^{d}$ these are exponential functions.

In our setting, Choquet-Deny method is modified in the following way: for any minimal harmonic function $h$ and $u \in E$, we show that the function $x \mapsto h(x \star u)$ is super-harmonic and its harmonic component, in the Riesz decomposition, is given by $\gamma_{u} h$ for some constant $\gamma_{u}>0$. Assuming that the hitting probabilities of the random walk are slowly varying, we prove that $\gamma_{u}=1$. In this way, we get some functional relations proving that any harmonic function $h$ of $(X(t))$ is superharmonic for the ladder height process $\left(H_{n}\right)$ with a potential, in the Riesz decomposition, component given by $h(e) V$ and a harmonic component $\tilde{h}$ given by

$$
\tilde{h}(x)=\lim _{n \rightarrow \infty} \mathbb{E}_{x}\left(h\left(H_{n}\right), \mathcal{T}_{\vartheta}>n\right), \quad x \in E .
$$

Using this result together with the results obtained for the renewal function $V$, we conclude that any harmonic function $h$ of $(X(t))$ is 
- proportional to the renewal function $V$ if the exit time $\tau_{\vartheta}$ is not integrable;

- bounded below by $h(e) V$, so that the difference $h-h(0) V$ is a non trivial harmonic function of the ladder height process $\left(H_{n}\right)$, otherwise.

Our results are applied next for a random walk $(X(t))$ on $\mathbb{Z}^{d}$ killed up on the time of the first exit from the closure of some open convex cone having a vertex at 0 . We show that for a centered irreducible random walk, or more generally, if generating function of the distribution of the jump of the random walk achieves its strict minimum at the origin $0 \in \mathbb{R}^{d}$, the hitting probabilities are then slowly varying. This result is obtained by using large deviation estimates for truncated random walks.

The functional relations obtained for the harmonic functions provide a probabilistic interpretation of the values $h(x) / h(x \star u)$ in terms of the corresponding $h$-transform: if the transition probabilities of the random walk $(X(t))$ satisfy the identity $p(x \star u, y \star u)=p(x, y)$ for all $x, y, u \in E$ and the hitting probabilities are slowly varying, then for any harmonic function $h$ and $x, u \in E$, the probability that the $h$-transform process starting at $x \star u$ never exits from the set $E \star u$ is equal to $h(x) / h(x \star u)$. This property could be of interest in view of applications to random walks in $\mathbb{Z}^{d}$ conditioned to stay in some convex cone. Denisov and Wachtel 6] have proved that, for a centered random walk in a general cone, under some moment conditions on the distribution of the jumps, the sequence of ratios (1.1) converges to some harmonic function $\mathcal{V}$. The corresponding random walk conditioned on the sequence of events $\left(\left\{\tau_{\vartheta}>n\right\}\right)$ to stay in the cone, is in this case the $\mathcal{V}$-transform of the original random walk $(X(t))$.

Remark finally that, in higher dimensions, a new phenomenon occurs: in contrast to a centered one dimensional random walk, the exit time $\tau_{\vartheta}$ can be integrable and, in this case, the limiting harmonic function

$$
\mathcal{V}=\lim _{n} \mathbb{P} .\left(\tau_{\vartheta}>n\right) / \mathbb{P}_{0}\left(\tau_{\vartheta}>n\right)
$$

obtained by Denisov and Wachtel [6] is not equal to the renewal function $V$.

\section{Preliminaries}

Before formulating our results we recall well known results in fluctuation theory for one dimensional random walks. The classical references are here the books of Spitzer [8] and Feller 9].

Denote by $(S(t))$ a homogeneous irreducible and aperiodic random walk on $\mathbb{Z}$ and let $(X(t))$ be a copy of $(S(t))$ killed up on the first exit from $\mathbb{Z}_{+}=\{k \in \mathbb{Z}: k \geq 0\}$. The strict descending ladder height process $\left(H_{k}, t_{k}\right)$ for the random walk $(S(t))$ is defined by

$$
t_{0}=0, \quad t_{k+1}=\inf \left\{n>t_{k}: S(n)<S\left(t_{k}\right)\right\} \quad \text { and } \quad H_{k}=S\left(t_{k}\right), \quad k \geq 0 .
$$

$\left(H_{k}\right)$ is therefore a substochastic random walk on $\mathbb{Z}$ with transition probabilities $p_{H}(x, y)=$ $\mathbb{P}_{0}(S(\tau)=y-x)$ where $\tau=\inf \{t>0: S(t)<0\}$. The random walk $\left(H_{k}\right)$ is stochastic if $\mathbb{P}_{0}$-almost surely $\tau<\infty$. If $\mathbb{P}_{0}(\tau=\infty)>0$ it is convenient to introduce an absorbing state $\vartheta$ for $\left(H_{k}\right)$ by letting

$$
p_{H}(x, \vartheta)=1-\sum_{y \in \mathbb{Z}_{+}} p_{H}(x, y), \quad x \in \mathbb{Z}_{+} .
$$

The renewal function $V$ associated with $\left(H_{k}\right)$ is then defined by

$$
V(x)=\sum_{k=0}^{\infty} \mathbb{P}_{x}\left(H_{k} \in \mathbb{Z}_{+}\right), \quad x \in \mathbb{Z}_{+}
$$

or equivalently,

$$
V(x)=\mathbb{E}_{x}(\mathcal{T}), \quad x \in \mathbb{Z}_{+} .
$$

where $\mathcal{T}$ denotes the first time when the Markov chain $H_{n}$ exits from the set $\mathbb{Z}_{+}$:

$$
\mathcal{T}=\inf \left\{n>0: H_{n} \notin \mathbb{Z}_{+}\right\}=\inf \left\{n>0: H_{n} \in\{\vartheta\} \cup \mathbb{Z}_{-}\right\}, \quad \mathbb{Z}_{-}=\mathbb{Z} \backslash \mathbb{Z}_{+} .
$$


If $\mathbb{P}_{0}(\tau=+\infty)>0$, i.e. when the random walk $(S(t))$ drifts to $+\infty$, the function $V$ satisfies the equality

$$
V(x)=\mathbb{P}_{x}(\tau=+\infty) / \mathbb{P}_{0}(\tau=+\infty), \quad \forall x \in \mathbb{Z}_{+} .
$$

If the reflected random walk $(-S(t))$ drifts to $+\infty$, i.e. when $(S(t))$ drifts to $-\infty$, the function $x \rightarrow \mathbb{E}_{x}(\tau)$ is finite everywhere on $\mathbb{Z}$ and

$$
V(x)=\mathbb{E}_{x}(\tau) / \mathbb{E}_{0}(\tau), \quad \forall x \in \mathbb{Z}_{+} .
$$

Finally, the random walk $(S(t))$ is called oscillating if it neither drifts to $+\infty$ nor to $+\infty$. In this case, $\mathbb{E}_{x}(\tau)=+\infty$ and

$$
\lim _{n \rightarrow \infty} \mathbb{P}_{x}(\tau>n) / \mathbb{P}_{0}(\tau>n)=V(x), \quad \forall x \in \mathbb{Z}_{+} .
$$

Moreover, if a random walk $(S(t))$ either drifts to $+\infty$ or oscillates, the function $V$ is harmonic for the killed random walk $(X(t))$ and any harmonic function of $(X(t))$ is proportional to $V$.

The purpose of the present paper is to extend the notion of the ladder height process and to obtain similar results for random walks in semi-groups and in particular, for centered random walks in $\mathbb{Z}^{d}$ killed up on the first exit from some convex cone.

\section{MAin Results}

We begin our analysis with an application of the Choquet-Deny theory for a substochastic Markov chain $(X(t))$ on a countable set $E$ with transition probabilities $p(x, y)$, $x, y \in E$. The Markov chain $(X(t))$ is assumed to satisfy following conditions:

(A0) $(X(t))$ is irreducible and transient on $E$,

(A1) the set of states $E$ of $(X(t))$ is included to some group $(\mathcal{E}, \star)$;

(A2) there is $u \in E$ such that

$$
E \star u \subset E
$$

and for any $x, y \in E$,

$$
p(x \star u, y \star u) \geq p(x, y)
$$

The Markov chain $(X(t))$ being substochastic, it is convenient to introduce an additional absorbing state $\vartheta$ by letting

$$
p(x, \vartheta)=1-\sum_{y \in E} p(x, y), \quad x \in E .
$$

$(X(t))$ is then a Markov chain on $E \cup\{\vartheta\}$ stopped when hitting the state $\vartheta$. We denote

$$
\tau_{\vartheta}=\inf \{t \geq 1: X(t)=\vartheta\} .
$$

The Green function $G(x, y)$ of the Markov chain $(X(t))$ is defined by

$$
G(x, y)=\sum_{t=0}^{\infty} \mathbb{P}_{x}(X(t)=y)=\sum_{t=0}^{\infty} \mathbb{P}_{x}\left(X(t)=y, t<\tau_{\vartheta}\right), \quad x, y \in E,
$$

and the hitting probabilities are denoted by

$$
Q(x, y)=\mathbb{P}_{x}(X(t)=y \text { for some } 0<t<+\infty), \quad x, y \in E .
$$

For a non-negative function $\varphi: E \rightarrow \mathbb{R}_{+}$, we let

$$
G \varphi(x)=\sum_{y \in E} G(x, y) \varphi(y), \quad x \in E,
$$

and

$$
P \varphi(x)=\mathbb{E}_{x}(\varphi(X(1)))=\sum_{y \in E} p(x, y) \varphi(y), \quad x \in E,
$$


It is convenient moreover to introduce two operators $\varphi \rightarrow T_{u} \varphi$ and $\varphi \rightarrow A_{u} \varphi$ on the set of non-negative functions $\left\{\varphi: E \rightarrow \mathbb{R}_{+}\right\}$, by letting

$$
T_{u} \varphi(x)=\varphi(x \star u), \quad x \in E,
$$

and

$$
A_{u} \varphi(x)=\sum_{y \in E} a_{u}(x, y) \varphi(y), \quad x \in E
$$

with

$$
a_{u}(x, y)= \begin{cases}p(x \star u, y)-p\left(x, y \star u^{-1}\right), & \text { if } y \in E \star u, \\ p(x \star u, y) & \text { otherwise. }\end{cases}
$$

Remark that under the hypotheses (A1) and (A2), $a_{u}(x, y) \geq 0$ for all $x, y \in E$. For any non-negative function $\varphi: E \rightarrow \mathbb{R}_{+}$, the function $A_{u} \varphi: E \rightarrow \mathbb{R}_{+} \cup\{+\infty\}$ is therefore well defined and

$$
T_{u} P \varphi=P T_{u} \varphi+A_{u} \varphi .
$$

Recall that for a Markov chain $(X(t))$, a non-zero positive function $h: E \rightarrow \mathbb{R}_{+}$is called harmonic (respectively super harmonic) if $P h=h$ (respectively $P h \leq h$ ). A function $g: E \rightarrow \mathbb{R}_{+}$is called potential for $(X(t))$ if $g=G \varphi$ with some non-negative function $\varphi: E \rightarrow \mathbb{R}_{+}$. Such a function $\varphi: E \rightarrow \mathbb{R}_{+}$satisfies then the following relation

$$
\varphi=g-P g .
$$

Any potential function is super harmonic, and by the Riesz decomposition theorem, any super harmonic function $f$ is equal to a sum of a harmonic function $h=\lim _{n} P^{n} f$ and a potential function $g=G \varphi$ with $\varphi=(\mathbb{I}-P) f$, see for instance Woess [25].

We extend any harmonic or super harmonic function $h$ on $E \cup\{\vartheta\}$ by letting

$$
h(\vartheta)=0 .
$$

1 denotes throughout this paper the identity constant function, $1(x)=1$ for all $x \in E$, and $\mathbb{I}$ denotes the identity operator $: \mathbb{I} \varphi=\varphi$ for any function $\varphi: E \rightarrow \mathbb{R}$.

Definition 3.1. We will say that the hitting probabilities of the random walk $(X(t))$ are slowly varying along the element $u \in E$ if uniformly on $x \in E$,

$$
\lim _{n \rightarrow \infty} \frac{1}{n} \log Q\left(x, x \star u^{\star n}\right)=\lim _{n \rightarrow \infty} \frac{1}{n} \log Q\left(x \star u^{\star n}, x\right)=0,
$$

where $u^{\star 1}=u$ and $u^{\star(n+1)}=u^{\star n} \star u$ for $n \geq 1$.

Our first result is the following statement.

Theorem 1. Suppose that (AO)-(A2) are satisfied and let for the given $u \in E$, the hitting probabilities of $(X(t))$ be slowly varying along $u$. Then any harmonic function $h$ satisfies the following relations

$$
h(y \star u)=h(y)+G A_{u} h(y), \quad \forall y \in E .
$$

The proof of this result uses the arguments of Choquet-Deny theory and is given in Section [5. In Section 4] we apply our results for homogeneous random walks in $\mathbb{Z}^{d}$ killed up on the time of the first exit from some general cone. The results of this section show that under quite general assumptions on the cone, centered random walks satisfy (3.2).

The functional relations (3.3) are the key point of our approach. Before formulating our next results, we rewrite these relations for the case when (A2) holds with the equality and we obtain a probabilistic interpretation of (3.3) in terms of the corresponding $h$-transform. For this it is convenient to introduce the first time when the process $(X(t))$ exits from $E \star u$ :

$$
\eta_{u}=\inf \{t \geq 1: X(t) \notin E \star u\} .
$$


Recall that for a given non-zero harmonic function $h$ of $(X(t))$, the $h$-transform $\left(X_{h}(t)\right)$ of the process $(X(t))$ is defined as a Markov chain with transition probabilities

$$
p_{h}(x, y)=p(x, y) h(y) / h(x), x, y \in E .
$$

For any non-zero harmonic function $h$ of $(X(t))$, the transition matrix of the $h$-transform $\left(X_{h}(t)\right)$ is stochastic on $E$ and consequently, the process $\left(X_{h}(t)\right)$ does not exit from the set $E$. If the sequence of functions

$$
f_{n}(x)=\mathbb{P}_{x}\left(\tau_{\vartheta}>n\right) / \mathbb{P}_{e}\left(\tau_{\vartheta}>n\right), \quad x \in E
$$

converges to some non zero harmonic function $h: E \rightarrow \mathbb{R}_{+}$, then the random walk $(X(t))$ conditioned on the sequence of events $\left(\left\{\tau_{\vartheta}>n\right\}\right)$ to stay in $E$ is the corresponding $h$ transform of $(X(t))$, see for instance Bertoin and Doney [5].

Proposition 3.1. Suppose that (AO) and (A1) are satisfied and let for some $u \in E$, $E \star u \subset E$ and

$$
p(x \star u, y \star u)=p(x, y), \quad \forall x, y \in E .
$$

Then for any non negative function $h: E \rightarrow \mathbb{R}_{+}$,

$$
G A_{u} h(y)=\mathbb{E}_{y \star u}\left(h\left(X\left(\eta_{u}\right)\right), \eta_{u}<\tau_{\vartheta} \leq+\infty\right), \quad \forall y \in E .
$$

If moreover, the hitting probabilities of $(X(t))$ are slowly varying along the given element $u \in E$, then for any $x \in E$ and any non zero harmonic function $h$ of $(X(t))$, the quantity $h(x) / h(x \star u)$ is equal to the probability that the corresponding $h$ transform $\left(X_{h}(t)\right)$ starting at $x \star u$ never exits from the set $E \star u$.

Proof. Indeed, in this case, for any $y, z \in E$,

$$
G(y, z)=\sum_{t=0}^{\infty} \mathbb{P}_{y \star u}\left(X(t)=z \star u, \eta_{u}>t\right)
$$

and according to the definition of the matrix $A_{u}$,

Hence,

$$
a_{u}(x, y)= \begin{cases}0, & \text { if } y \in E \star u, \\ p(x \star u, y) & \text { otherwise }\end{cases}
$$

$$
\begin{aligned}
G A_{u} h(y) & =\sum_{z, z^{\prime} \in E} G(y, z) a_{u}\left(z, z^{\prime}\right) h\left(z^{\prime}\right) \\
& =\sum_{z \in E} \sum_{z^{\prime} \in E \backslash(E \star u)} \sum_{t=0}^{\infty} \mathbb{P}_{y \star u}\left(X(t)=z \star u, t<\eta_{u}\right) p\left(z \star u, z^{\prime}\right) h\left(z^{\prime}\right) \\
& =\mathbb{E}_{y \star u}\left(h\left(X\left(\eta_{u}\right)\right), \eta_{u}<\tau_{\vartheta} \leq+\infty\right) .
\end{aligned}
$$

The first assertion of Proposition 3.1 is therefore proved. The second assertion is a consequence of Theorem 1 To get this assertion, it is sufficient to notice that by (3.3) and (3.5), the probability that the $h$-transform $\left(X_{h}(t)\right)$ starting at $x \star u$ ever exits from the set $E \star u$ is equal to

$$
\frac{1}{h(x \star u)} \mathbb{E}_{x \star u}\left(h\left(X\left(\eta_{u}\right)\right) ; \eta_{u}<\tau_{\vartheta} \leq+\infty\right)=1-h(x) / h(x \star u) .
$$

Now we introduce an analogue of the ladder height process $\left(H_{n}\right)$ and extend the result of the classical fluctuation theory formulated in the previous section. From now on the random walk $(X(t))$ is assumed to satisfy the following conditions:

(B1) the state set $E$ of $(X(t))$ is included to some group $(\mathcal{E}, \star)$ and $(E, \star)$ is a semi-group with an identity element $e$; 
(B2) for any $x, y, u \in E$,

$$
p(x \star u, y \star u) \geq p(x, y),
$$

Remark that under the above assumptions, for any $u \in E$, the previous conditions (A1) and (A2) are satisfied and consequently, the operator $A_{u}$ on the set of non-negative functions $\left\{\varphi: E \rightarrow \mathbb{R}_{+}\right\}$is well defined.

To introduce the ladder Markov chain $\left(H_{n}\right)$ we need the following lemma.

Lemma 3.1. Under the hypotheses (A0), (B1) and (B2), the matrix

$$
P_{H}=\left(p_{H}(x, y), x, y \in E\right)
$$

with

$$
p_{H}(x, y)=G A_{x} 1_{\{y\}}(e)=\sum_{z \in E} G(e, z) a_{x}(z, y), \quad x, y \in E,
$$

is substochastic.

Proof. Indeed, the coefficients of the matrix $P_{H}$ are non-negative and for any $x \in E$

$$
\begin{aligned}
P_{H} 1(x) & =G A_{x} 1(e)=\lim _{n \rightarrow \infty} \sum_{k=0}^{n} P^{k} A_{x} 1(e)=\lim _{n \rightarrow \infty} \sum_{k=0}^{n} P^{k}\left(T_{x} P-P T_{x}\right) 1(e) \\
& =\lim _{n \rightarrow \infty}\left(\sum_{k=0}^{n} P^{k} T_{x} P 1(e)-\sum_{k=1}^{n+1} P^{k} T_{x} 1(e)\right)
\end{aligned}
$$

The above relation combined with the equality $T_{x} 1=1$ implies that

$$
\begin{aligned}
P_{H} 1(x) & =\lim _{n \rightarrow \infty}\left(\sum_{k=0}^{n} P^{k} T_{x} P 1(e)-\sum_{k=1}^{n+1} P^{k} 1(e)\right) \\
& =T_{x} P 1(e)-\lim _{n \rightarrow \infty}\left(\sum_{k=1}^{n} P^{k}\left(I d-T_{x} P\right) 1(e)+P^{n+1} 1(e)\right) .
\end{aligned}
$$

Since $P^{n+1} 1(e) \geq 0$ and

$$
P^{k}\left(I d-T_{x} P\right) 1(e)=\sum_{y \in E} \mathbb{P}_{e}(X(k)=y)\left(1-\sum_{z \in E} p(y \star x, z)\right) \geq 0,
$$

from the last relation it follows that $P_{H} 1(x) \leq T_{x} P 1(e) \leq 1$.

Definition 3.2. The ladder height process relative to the Markov chain $(X(t))$ is defined as a discrete time Markov chain $\left(H_{n}\right)$ on $E$ with transition matrix $P_{H}$.

The Markov chain $\left(H_{n}\right)$ being sub-stochastic on $E$, we introduce an additional absorbing state $\vartheta$. Without any restriction of generality we can keep the same notation of the additional absorbing state as for the random walk $(X(t))$. We let

$$
p_{H}(x, \vartheta)=1-\sum_{y \in E} p_{H}(x, y), \quad \forall x \in E .
$$

Before formulating our next result let us give another equivalent definition of the ladder Markov chain $\left(H_{n}\right)$ in a particular case, when (B2) holds with the equality. The following statement is a straightforward consequence of Proposition 3.1

Proposition 3.2. Suppose that (AO) and (B1) are satisfied and let

$$
p(x \star u, y \star u)=p(x, y), \quad \forall u, x, y \in E .
$$

Then for a sequence of random times $\left(t_{n}\right)$ defined for $n \in \mathbb{N}$ by

$$
t_{0}=0, \quad \text { and } \quad t_{k+1}= \begin{cases}\inf \left\{n>t_{k}: X(n) \notin E \star X\left(t_{k}\right)\right\}, & \text { if } t_{k}<+\infty, \\ +\infty & \text { otherwise, }\end{cases}
$$


in distribution

$$
H_{k}= \begin{cases}X\left(t_{k}\right) & \text { if } t_{k}<\infty \\ \vartheta, & \text { otherwise }\end{cases}
$$

Now we extend the notion of the renewal function $V: E \cup\{\vartheta\} \rightarrow \mathbb{R}_{+} \cup\{+\infty\}$. Denote $\mathcal{T}_{\vartheta}=\inf \left\{n \geq 0: H_{n}=\vartheta\right\}$ and let

$$
V(\vartheta)=0 \quad \text { and } \quad V(x)=\mathbb{E}_{x}\left(\mathcal{T}_{\vartheta}\right), \quad x \in E .
$$

Our next result proves that this function is finite and satisfies the properties similar to those of the renewal function for a one-dimensional random walk. Recall that under (A0), the Markov chain $(X(t))$ is irreducible in $E$. The function $x \rightarrow \mathbb{E}_{x}\left(\tau_{\vartheta}\right)$ is therefore either finite in $E$, or infinite everywhere in $E$.

Theorem 2. Under the hypotheses (A0), (B1) and (B2) the following assertions hold:

(i) The ladder height process $\left(H_{n}\right)$ is transient on $E$.

(ii) The function $V$ is finite and satisfies the following relations :

$$
V(e)=1
$$

and

$$
V(x \star u)=V(x)+G A_{u} V(x), \quad \forall x, u \in E .
$$

(iii) If $\mathbb{E} .\left(\tau_{\vartheta}\right)=+\infty$, the function $V$ is harmonic for the Markov chain $(X(t))$ and for any $x \in E$,

$$
\lim _{n \rightarrow \infty} \mathbb{P}_{x}\left(\tau_{\vartheta}>n\right) / \mathbb{P}_{e}\left(\tau_{\vartheta}>n\right)=V(x) .
$$

(iv) If $\mathbb{E} .\left(\tau_{\vartheta}\right)<+\infty$, the function $V$ is potential for the Markov chain $(X(t))$ and for any $x \in E$,

$$
V(x)=\mathbb{E}_{x}\left(\tau_{\vartheta}\right) / \mathbb{E}_{e}\left(\tau_{\vartheta}\right) \leq \liminf _{n \rightarrow \infty} \mathbb{P}_{x}\left(\tau_{\vartheta}>n\right) / \mathbb{P}_{e}\left(\tau_{\vartheta}>n\right)
$$

The proof of this theorem is given in section 6 .

Remark that under the hypotheses of Theorem 1 and Theorem 2 , by (3.12) and (3.3), the renewal function $V$ satisfies the same functional relations as any harmonic function $h$. This is the key point of the proof of our next result.

From now on the Green's function of the ladder height process $\left(H_{n}\right)$ will be denoted by

$$
G_{H}(x, y)=\sum_{n=0}^{\infty} \mathbb{P}_{x}\left(H_{n}=y\right), \quad x, y \in E .
$$

For $n \geq 1$ and a non negative function $\varphi: E \rightarrow \mathbb{R}_{+}$we introduce

$$
P_{H}^{n} \varphi(x)=\mathbb{E}_{x}\left(\varphi\left(H_{n}\right) ; \mathcal{T}_{\vartheta}>n\right)
$$

and

$$
G_{H} \varphi(x)=\sum_{n=0}^{\infty} P_{H}^{n} \varphi(x)=\sum_{n=0}^{\infty} \mathbb{E}_{x}\left(\varphi\left(H_{n}\right) ; \mathcal{T}_{\vartheta}>n\right) .
$$

Theorem 3. Suppose that the conditions (A0), (B1) and (B2) are satisfied and let the hitting probabilities of the random walk $(X(t))$ be slowly varying along every $u \in E$. Then any harmonic function $h$ of $(X(t))$ is super harmonic for the ladder height process $\left(H_{n}\right)$ and

$$
h(x)=h(e) V(x)+\tilde{h}(x), \quad \forall x \in E,
$$

with a harmonic, for the ladder height process $\left(H_{n}\right)$, function

$$
\tilde{h}(x)=\lim _{n \rightarrow \infty} \mathbb{E}_{x}\left(h\left(H_{n}\right) ; \mathcal{T}_{\vartheta}>n\right), \quad \forall x \in E .
$$


If moreover $\mathbb{E} .\left(\tau_{\vartheta}\right)=+\infty$, then for any harmonic function $h$, the function (3.16) is equal to zero and $h$ is proportional to $V$. Otherwise, for any harmonic function $h$, the function (3.16) is non trivial.

The proof of this theorem is given in section 7 .

\section{Application to a homogeneous Random Walk}

In this section, we apply our results for a homogeneous random walk in $Z^{d}$ killed up on the time of the first exit from a convex cone.

Consider a probability measure $\mu$ on $\mathbb{Z}^{d}$ and let $(X(t))$ be a substochastic random walk on $E$ with transition probabilities $\mathbb{P}_{x}(X(1)=y)=p(x, y)=\mu(y-x), x, y \in E$. Such a random walk is a copy of a homogeneous random walk $(S(t))$ in $\mathbb{Z}^{d}$ with transition probabilities $p(x, y)=\mu(y-x)$, killed up on the first exit from $E$. The additional absorbing state $\vartheta$ is then identified with the set $\mathbb{Z}^{d} \backslash E$ : for every $x \in E$ we let

$$
p(x, \vartheta)=1-\sum_{y \in E} \mu(y-x)
$$

We will assume that

(C0) the random walk $(X(t))$ is irreducible and transient on $E$;

(C1) $0 \in E$ and for any $x, u \in E, \quad x+u \in E$.

Remark that under the above assumptions, the conditions (A0), (B1) and (B2) of the previous section are satisfied and consequently, the ladder process $\left(H_{n}\right)$ relative to the random walk $(X(t))$ is well defined. Moreover, in this case, (B2) holds with the equality, and hence, by Proposition 3.2 for a sequence of random times $\left(t_{n}\right)$ defined by

$$
t_{0}=0, \quad \text { and } \quad t_{k+1}= \begin{cases}\inf \left\{n>t_{k}: X(n) \notin E+X\left(t_{k}\right)\right\} & \text { if } t_{k}<\infty \\ +\infty & \text { otherwise }\end{cases}
$$

in distribution

$$
H_{k}= \begin{cases}X\left(t_{k}\right) & \text { if } t_{k}<\infty \\ \vartheta, & \text { otherwise }\end{cases}
$$

Consider now the stopping times $\mathcal{T}_{\vartheta}=\inf \left\{n>0: H_{n}=\vartheta\right\}$ and $\tau_{\vartheta}=\inf \{n>0: X(n)=$ $\vartheta\}$, and let for $x \in E, \eta_{x}=\inf \{t>0: X(t) \notin E+x\}$. Recall that the renewal function $V: E \cup\{\vartheta\} \rightarrow \mathbb{R}_{+}$is defined by $V(x)=\mathbb{E}_{x}\left(\mathcal{T}_{\vartheta}\right)$ with $V(\vartheta)=0$. The following statement is a consequence of Theorem 2 .

Corollary 4.1. Under the hypotheses (CO) and (C1) the following assertions hold.

(i) The ladder height process $\left(H_{n}\right)$ is transient on $E$.

(ii) The function $V$ is finite with $V(0)=1$ and satisfies the following relations

$$
V(x+u)=V(x)+\mathbb{E}_{x+u}\left(V\left(X\left(\eta_{u}\right)\right), \eta_{u}<\tau_{\vartheta} \leq \infty\right), \quad \forall x, u \in E .
$$

(iii) If $\mathbb{E} .\left(\tau_{\vartheta}\right)=+\infty$, the function $V$ is harmonic for the Markov chain $(X(t))$ and for any $x \in E$,

$$
\lim _{n \rightarrow \infty} \mathbb{P}_{x}\left(\tau_{\vartheta}>n\right) / \mathbb{P}_{0}\left(\tau_{\vartheta}>n\right)=V(x)
$$

(iv) If $\mathbb{E} .\left(\tau_{\vartheta}\right)<+\infty$, the function $V$ is potential for the Markov chain $(X(t))$ and for any $x \in E$,

$$
V(x)=\mathbb{E}_{x}\left(\tau_{\vartheta}\right) / \mathbb{E}_{0}\left(\tau_{\vartheta}\right) \leq \liminf _{n \rightarrow \infty} \mathbb{P}_{x}\left(\tau_{\vartheta}>n\right) / \mathbb{P}_{0}\left(\tau_{\vartheta}>n\right)
$$

Proof. The assertions (i), (iii) and (iv) are proved by the corresponding assertions of Theorem 2, The assertion (ii) follows from the second assertion of Theorem 2 combined with Proposition 3.1

Suppose now that 
$(\mathrm{C} 0$ ') the random walk $(X(t))$ is transient on $E$ and satisfies the following communication condition : there are $\kappa>0$ and a finite set $\mathcal{E}_{0} \subset \mathbb{Z}^{d}$ such that

(a) $\mu(x)>0$ for all $x \in \mathcal{E}_{0}$;

(b) for any $x \neq y, x, y \in E$ there exists a sequence $x_{0}, x_{1}, \ldots, x_{n} \in E$ with $x_{0}=$ $x, x_{n}=y$ and $n \leq \kappa|y-x|$ such that $x_{j}-x_{j-1} \in \mathcal{E}_{0}$ for all $j \in\{1, \ldots, n\}$.

(C1') there is an open convex cone $\mathcal{C}$ in $\mathbb{R}^{d}$ with a vertex in the origin $0 \in \mathbb{R}^{d}$, such that $E=\overline{\mathcal{C}} \cap \mathbb{Z}^{d}$, where $\overline{\mathcal{C}}$ denotes the closure of $\mathcal{C}$ in $\mathbb{R}^{d}$.

(C2) the jump generating function $R: \mathbb{Z}^{d} \rightarrow \mathbb{R} \cup\{+\infty\}$ defined by

$$
R(\alpha)=\sum_{x \in \mathbb{Z}^{d}} \mu(x) e^{\langle\alpha, x\rangle}, \quad \alpha \in \mathbb{R}^{d}
$$

achieves its minimum at $0 \in \mathbb{R}^{d}$ and for any $\alpha \in \mathbb{R}^{d} \backslash\{0\}, R(\alpha)>R(0)=1$.

Remark that the last condition is satisfied for integrable and centered random walks, when

$$
\sum_{x \in \mathbb{Z}^{d}} x \mu(x)=0
$$

and also for non integrable random walks if $R(\alpha)=+\infty$ for all $\alpha \neq 0$.

Our next result proves that under the above assumptions, the hitting probabilities

$$
Q(z, y)=\mathbb{P}_{z}\left(X(t)=y \text { for some } t<\tau_{\vartheta}\right), \quad x, y \in E .
$$

are slowly varying along any vector $u \in E$.

Proposition 4.1. Under the hypotheses (C0'), (C1') and (C2), for any $u \in E$ and uniformly on $x \in E$,

$$
\lim _{n \rightarrow \infty} \frac{1}{n} \log Q(x, x+n u)=\lim _{n \rightarrow \infty} \frac{1}{n} \log Q(x+n u, x)=0,
$$

The proof of this proposition is given in Section 9 When combined with Theorem 1 and Theorem 3 this result provides the following statement.

Corollary 4.2. Under the hypotheses (C0'), (C1') and (C2), any harmonic function $h$ of $(X(t))$ is super harmonic for the ladder height process $\left(H_{n}\right)$ and satisfies the relation

$$
h(x+u)=h(x)+\mathbb{E}_{x+u}\left(h\left(X\left(\eta_{u}\right)\right), \eta_{u}<\infty\right), \quad \forall x, u \in E,
$$

and

$$
h=h(0) V+\tilde{h}
$$

with a harmonic, for the ladder height process $\left(H_{n}\right)$, function

$$
\tilde{h}(x)=\lim _{n \rightarrow \infty} \mathbb{E}_{x}\left(h\left(X\left(t_{n}\right)\right), t_{n}<\infty\right) \geq 0 .
$$

If moreover $\mathbb{E}_{x}\left(\tau_{\vartheta}\right)=+\infty$, then for any harmonic function $h$, the function (4.4) is equal to zero and $h$ is proportional to $V$. Otherwise, the function (4.4) is non trivial.

A straightforward consequence of Corollary 4.1 and Corollary 4.2 is the following statement.

Corollary 4.3. Suppose that the conditions (C0'), (C1') and (C2) are satisfied, the steps of the random walk $(S(t))$ are integrable:

$$
\sum_{x \in \mathbb{Z}^{d}}|x| \mu(x)<+\infty
$$

and let

$$
m=\sum_{x \in \mathbb{Z}^{d}} x \mu(x) \neq 0 \quad \text { and } \quad m \in \mathcal{C} .
$$

Then for any $x \in E, V(x)=\mathbb{P}_{x}\left(\tau_{\vartheta}=+\infty\right) / \mathbb{P}_{0}\left(\tau_{\vartheta}=+\infty\right)$ and any harmonic function $h$ of the killed random walk $(X(t))$ is proportional to $V$. 
Proof. If the steps of the random walk $(S(t))$ are integrable, for any $x \in \mathbb{Z}^{d}, \mathbb{P}_{x^{-}}$almost surely

$$
\lim _{n \rightarrow \infty} S(n) / n=m \in \mathcal{C} .
$$

The cone $\mathcal{C}$ being open, from this it follows that for any $x \in E, \mathbb{P}_{x}$-almost surely

$$
\sup \{n \in \mathbb{N}: S(n) \notin \mathcal{C}\}<\infty
$$

and consequently, $\mathbb{P}_{x}$-almost surely

$$
\sup \{N: S(n)+N m \notin \mathcal{C} \text { for some } n \in \mathbb{N}\}<\infty .
$$

This proves that for any $x \in E$ there is $N \geq 0$ such that

$$
P_{x}(S(n)+N m \in \mathcal{C}, \forall n \geq 0)>0 .
$$

For $y \in(C+N m) \cap \mathbb{Z}^{d}$, one gets therefore

$$
\begin{aligned}
\mathbb{P}_{x+y}(S(n) \in \mathcal{C}, \forall n \geq 0) & =P_{x}(S(n)+y \in \mathcal{C}, \forall n \geq 0) \\
& \geq P_{x}(S(n)+N m \in \mathcal{C}, \forall n \geq 0)>0
\end{aligned}
$$

because $C-N m \subset C-y$, and consequently,

$$
\mathbb{P}_{x+y}\left(\tau_{\vartheta}=+\infty\right) \geq \mathbb{P}_{x+y}(S(n) \in \mathcal{C}, \forall n \geq 0)>0
$$

The random walk $\left(X(t)\right.$ being irreducible on $E=\mathbb{Z}^{d} \cap E$, from this it follows that the function $x \rightarrow \mathbb{P}_{x}\left(\tau_{\vartheta}=+\infty\right)$ is non zero everywhere on $E$ and in prticular, the exit time $\tau_{\vartheta}$ is non-integrable. Using Corollary 4.1 we conclude therefore that the function $V$ is harmonic for the killed random walk $(X(t))$,

$$
V(x)=\lim _{n \rightarrow \infty} \mathbb{P}_{x}\left(\tau_{\vartheta}>n\right) / \mathbb{P}_{0}\left(\tau_{\vartheta}>n\right)=\mathbb{P}_{x}\left(\tau_{\vartheta}+\infty\right) / \mathbb{P}_{0}\left(\tau_{\vartheta}=\infty\right),
$$

for any $x \in E$ and by Corollary 4.2 any harmonic function $h$ of $(X(t))$ is proportional to $V$.

Recall that for centered random walks, i.e. when

$$
\sum_{x \in \mathbb{Z}^{d}} x \mu(x)=0
$$

under some additional moment conditions on the jump distribution $\mu$, the asymptotic of the tail distribution of $\tau_{\vartheta}$ were obtained by Denisov and Wachtel [6]. They construct a harmonic function $\mathcal{V}$ by using the harmonic function of the corresponding diffusion approximation, and they proved that for any initial position $x$,

$$
\mathbb{P}_{x}\left(\tau_{\vartheta}>n\right) \sim \kappa \mathcal{V}(x) n^{-p / 2}
$$

with some constant $\kappa>0$ and some $p>0$ depending on the cone and also on the covariance matrix of the process. This result provides the pointwise convergence

$$
\lim _{n \rightarrow \infty} \mathbb{P}_{x}\left(\tau_{\vartheta}>n\right) / \mathbb{P}_{0}\left(\tau_{\vartheta}>n\right)=\mathcal{V}(x), \quad x \in E .
$$

Under the hypotheses of the paper [6], the random walk $(X(t))$ conditioned on the events $\left\{\tau_{\vartheta}>n\right\}$ to stay in the cone, is therefore the $\mathcal{V}$-transform of $(X(t))$, see for instance 5 for more details. Unfortunately, the representation of the function $\mathcal{V}$ obtained in [6] is rather implicit and the probabilities related to the corresponding conditioned random walk are difficult to investigate. With our approach, under the hypotheses (C0') and (C1') together with the hypotheses of [6], by Corollary 4.1] and 4.2 we obtain for the harmonic function $\mathcal{V}$ constructed in [6] the following properties :

- For any $x, u \in E$, the function $\mathcal{V}$ satisfies the equality

$$
\mathcal{V}(x+u)=\mathcal{V}(x)+\mathbb{E}_{x+u}\left(\mathcal{V}\left(X\left(\eta_{u}\right)\right), \eta_{u}<\infty\right)
$$

- For any $x, u \in E$, the quantity $\mathcal{V}(x) / \mathcal{V}(x+u)$ is equal to the probability that the random walk conditioned on the sequence of events $\left\{\tau_{\vartheta}>n\right\}$ to stay in $E$, starting at $x+u$ never exists from the set $E+u$. 
- In particular, for any $x \in E$, the quantity $1 / \mathcal{V}(x)$ is equal to the probability that the conditioned random walk starting at $x$ never exits from $E+x$,

- If $p \leq 2$, then the function $\mathcal{V}$ is identical to the renewal function $V$,

- If $p>2$, then $\mathcal{V}=V+\tilde{h}=\mathbb{E} .\left(\tau_{\vartheta}\right) / \mathbb{E}_{0}\left(\tau_{\vartheta}\right)+\tilde{h}$ with a non trivial harmonic for the ladder height process function $\tilde{h}=\lim _{n \rightarrow \infty} \mathbb{E} .\left(\mathcal{V}\left(X\left(t_{n}\right)\right)\right)$

Example. Consider a two dimensional irreducible random walk $X(t)=\left(X_{1}(t), X_{2}(t)\right)$ in $\mathbb{Z}_{+}^{2}$ killed upon the first exit from the positive quadrant $\mathbb{Z}_{+}^{2}$, with transition probabilities $\mathbb{P}_{x}(X(1)=y)=p(x, y)=\mu(y-x), x, y \in \mathbb{Z}_{+}^{2}$ such that

$$
\sum_{x \in \mathbb{Z}^{2}} x \mu(x)=0 .
$$

Assume moreover that

$$
\left.\sum_{x=\left(x_{1}, x_{2}\right) \in \mathbb{Z}^{2}} x_{1}^{2} \mu(x)=\sum_{x=\left(x_{1}, x_{2}\right) \in \mathbb{Z}^{2}} x_{2}^{2} \mu(x)=1 \quad \text { and } \sum_{x=\left(x_{1}, x_{2}\right) \in \mathbb{Z}^{2}} x_{1} x_{2} \mu(x)=\rho \in\right]-1,1[\text {. }
$$

In this case $p=\pi / \arccos (-\rho)$ (see example 3 of the paper [6]), and if

$$
\sum_{x \in \mathbb{Z}^{2}}|x|^{p} \mu(x)<+\infty
$$

then

$$
\mathbb{P}_{x}\left(\tau_{\vartheta}>n\right) \sim \kappa \mathcal{V}(x) n^{-\pi /(2 \arccos (-\rho))}, \quad x \in \mathbb{Z}_{+}^{2}
$$

Hence, the exit time $\tau_{\vartheta}=\inf \left\{n \geq 0: X(n) \notin \mathbb{Z}_{+}^{2}\right\}$ is integrable if and only if $\rho<0$. Using our results one gets therefore the following statements :

- if $\rho \geq 0$, then, up to multiplications by constants, the renewal function $V$ is the unique harmonic function for the Markov chain $(X(t))$, and for any $x \in \mathbb{Z}_{+}^{2}$,

$$
V(x)=\lim _{n \rightarrow \infty} \mathbb{P}_{x}\left(\tau_{\vartheta}>n\right) / \mathbb{P}_{0}\left(\tau_{\vartheta}>n\right)=\mathcal{V}(x)
$$

- if $\rho<0$, then the renewal function $V$ is potential for the Markov chain $(X(t))$,

$$
V(x)=\mathbb{E}_{x}\left(\tau_{\vartheta}\right) / \mathbb{E}_{0}\left(\tau_{\vartheta}\right)<\liminf _{n \rightarrow \infty} \mathbb{P}_{x}\left(\tau_{\vartheta}>n\right) / \mathbb{P}_{0}\left(\tau_{\vartheta}>n\right)=\mathcal{V}(x) \quad \forall x \in \mathbb{Z}_{+}^{2}
$$

where $\tilde{h}=\mathcal{V}-V$ is a non trivial harmonic for ladder height process function. Moreover, in a particular case, when $\rho=0$, the function $h: \mathbb{Z}_{+}^{2} \rightarrow \mathbb{R}_{+}$defined by

$$
h\left(x_{1}, x_{2}\right)=\left(x_{1}+1\right)\left(x_{2}+1\right), \quad x=\left(x_{1}, x_{2}\right) \in \mathbb{Z}_{+}^{2},
$$

is clearly harmonic for $(X(t))$. In this particular case one concludes therefore that $V=$ $\mathcal{V}=h$ and for any $x=\left(x_{1}, x_{2}\right), u=\left(u_{1}, u_{2}\right) \in \mathbb{Z}_{+}^{2}$, the probability that the random walk conditioned on the sequence of events $\left\{\tau_{\vartheta}>n\right\}$ to stay in $\mathbb{Z}_{+}^{2}$, starting at $x+u$ never exists from the set $\mathbb{Z}_{+}^{2}+u$ is equal to $\left(x_{1}+1\right)\left(x_{2}+1\right) /\left(\left(x_{1}+u_{1}+1\right)\left(x_{2}+u_{2}+1\right)\right)$.

\section{Proof of Theorem 1}

5.1. Preliminary results. We begin the proof of this theorem with the following lemma. It provides Harnack's inequality for super harmonic functions. This first result is classical and is given here for convenience of the reader to avoid a confusion with another types of Harnack inequalities, see for instance the book of Woess [25].

Lemma 5.1. For any super harmonic function $f: E \rightarrow \mathbb{R}_{+}$and $x, y \in E$,

$$
f(x) \geq Q(x, y) f(y) .
$$

Proof. Let $y \in E$ and denote by $\tau_{y}$ the first time when the Markov chain $(X(t))$ hits the state $y$ :

$$
\tau_{y}=\inf \{t \geq 0: X(t)=y\} .
$$

If the function $f: E \rightarrow \mathbb{R}_{+}$is super harmonic for $(X(t))$ then the sequence $(h(X(n)))$ is a super martingale relative to the natural filtration of $(X(t))$, and by the stopping time 
theorem, the sequence $\left(h\left(X\left(\min \left\{n, \tau_{y}\right\}\right)\right)\right)$ is also super martingale. This proves that for any super harmonic function $h: E \rightarrow \mathbb{R}_{+}, n \geq 0$ and $x, y \in E$,

$h(x) \geq \mathbb{E}\left(h\left(X\left(\min \left\{n, \tau_{y}\right\}\right)\right)\right)=h(y) \mathbb{P}_{x}\left(\tau_{y} \leq n\right)+\mathbb{E}\left(h(X(n)) ; \tau_{y}>n\right) \geq h(y) \mathbb{P}_{x}\left(\tau_{y} \leq n\right)$

Letting at the last inequality $n \rightarrow+\infty$, by monotone convergence theorem one gets (5.1).

Lemma 5.2. Suppose that (AO) - (A2) are satisfied and let there exists $\delta>0$ such that

$$
Q(x, x \star u) \geq \delta \quad \forall x \in E .
$$

Then the following assertions hold:

(a) for any super harmonic function $f$, the function $T_{u} f$ is also super harmonic and satisfies the inequality $\delta T_{u} f \leq f$;

(b) for any potential function $g$, the function $T_{u} g$ is also potential :

$$
T_{u} g=G \varphi \quad \text { with } \varphi=(\mathbb{I}-P) T_{u} g .
$$

(c) for any minimal harmonic function $h$, there exists $\gamma \geq 0$ such that for any $n \in \mathbb{N}$, $n \geq 1$, the function $T_{u}^{n} h-\gamma^{n} h$ is potential and

$$
T_{u}^{n} h-\gamma^{n} h=G A_{u^{\star n}} h=\sum_{k=1}^{n} \gamma^{k-1} T_{u}^{n-k} G A_{u} h
$$

Proof. Suppose that a function $f: E \rightarrow \mathbb{R}_{+}$is super harmonic. Then by (A2),

$$
\begin{aligned}
P T_{u} f(x) & =\sum_{y \in E} p(x, y) f(y \star u) \leq \sum_{y \in E} p(x \star u, y \star u) f(y \star u) \\
& \leq \sum_{z \in E} p(x \star u, z) f(z) \leq f(x \star u)=T_{u} f(x), \quad \forall x \in E .
\end{aligned}
$$

The function $T_{u} f$ is therefore also super harmonic. Moreover, from (5.2) it follows that

$$
\delta T_{u} f(x)=\delta f(x \star u) \leq Q(x, x \star u) f(x \star u), \quad \forall x \in E .
$$

and by (5.1),

$$
Q(x, x \star u) f(x \star u) \leq f(x), \quad \forall x \in E,
$$

from which it follows that $\delta T_{u} f \leq f$. The first assertion of our lemma is therefore proved.

To prove the second assertion, recall that every potential function is super harmonic, and a super harmonic function $g$ is potential if and only if $\lim _{n} P^{n} g=0$. For any potential function $g$, the first assertion of our lemma proves that the function $T_{u} g$ is super harmonic and

$$
\lim _{n} P^{n} T_{u} g(x) \leq \frac{1}{\delta} \lim _{n} P^{n} g(x)=0, \quad \forall x \in E .
$$

For a potential function $g$, the function $T_{u} g$ is therefore also potential: $T_{u} g=G \varphi$ with $\varphi=(\mathbb{I}-P) T_{u} g$.

To prove the third assertion of Lemma 5.2 we use the Riesz decomposition theorem. Suppose that $h$ is a minimal harmonic function. Then from the first assertion of our lemma it follows that for any $n \in \mathbb{N}$, the function $T_{u}^{n} h=T_{u^{\star n}} h$ is super harmonic and satisfies the inequality $\delta^{n} T_{u^{\star n}} h \leq h$. Using the Riesz decomposition theorem, we conclude that there exist a harmonic function $h_{n}=\lim _{k} P^{k} T_{u^{\star n}} h$ and a potential function $G \varphi_{n}$ such that

$$
T_{u^{\star n}} h=h_{n}+G \varphi_{n}
$$

The last relation combined with the inequality $\delta^{n} T_{u^{\star n}} h \leq h$, proves that $\delta^{n} h_{n} \leq h$. The harmonic function $h$ being minimal, from this it follows that $h_{n}=\gamma_{n} h$ for some $\gamma_{n} \geq 0$, and consequently,

$$
T_{u^{\star n}} h=\gamma_{n} h+G \varphi_{n} .
$$


By iterating the last equality with $n=1$, one gets

$$
T_{u \star n} h=T_{u}^{n} h=\gamma_{1}^{n} h+\sum_{k=1}^{n} \gamma_{1}^{k-1} T_{u}^{n-k} G \varphi_{1} .
$$

The second assertion of our lemma proves that the function

$$
\sum_{k=1}^{n} \gamma_{1}^{k-1} T_{u}^{n-k} G \varphi_{1}
$$

is potential as a sum of potential functions. Hence, by uniqueness of the Riesz decomposition, from (5.4) and (5.5) it follows that

$$
\gamma_{n}=\gamma_{1}^{n} \quad \text { and } \quad G \varphi_{n}=\sum_{k=1}^{n} \gamma_{1}^{k-1} T_{u}^{n-k} G \varphi_{1} .
$$

Finally, recall that $(\mathbb{I}-P) h=0$ and according to the definition of $A_{u^{\star n}}$,

$$
A_{u^{\star n}} h=T_{u^{\star n}} P h-P T_{u^{\star n}} h .
$$

Hence, from (5.4) one gets

$$
\varphi_{u^{\star n}}=(\mathbb{I}-P) T_{u^{\star n}} h=T_{u^{\star n}} P h-P T_{u^{\star n}} h=A_{u^{\star n}} h,
$$

and consequently, (5.3) holds with $\gamma=\gamma_{1}$. Lemma 5.2 is therefore proved.

Lemma 5.3. Under the hypotheses of Theorem 1, there exists $\delta>0$ for which (5.2) holds.

Proof. Indeed, from (3.2) it follows that there exist $c>0$ and $n>0, n \in \mathbb{N}$, such that

$$
Q\left(x, x \star u^{\star n}\right)>c \text { and } Q\left(x \star u^{\star(n-1)}, x\right)>c, \quad \forall x \in E .
$$

When combined with (A1), these inequalities imply that

$$
Q(x, x \star u) \geq Q\left(x, x \star u^{\star n}\right) Q\left(x \star u^{\star n}, x \star u\right) \geq Q\left(x, x \star u^{\star n}\right) Q\left(x \star u^{\star(n-1)}, x\right) \geq c^{2}
$$

for all $x \in E$. The last relation proves (5.2) with $\delta=c^{2}$

5.2. Proof of Theorem 1. Now we are ready to prove Theorem 1 For this it is sufficient to show that under the hypotheses of Theorem 1, every non-zero harmonic function $h$ satisfies (5.3) with $\gamma=1$. We begin our proof with the case when $h: E \rightarrow \mathbb{R}_{+}$is a minimal harmonic function for $(X(t))$.

Suppose that the conditions (A0)-(A2) are satisfied and let for some $u \in E$, (3.2) hold uniformly on $x \in E$. Then by Lemma 5.3 the inequalities (5.2) hold with some $\delta>0$, and consequently, by Lemma [5.2, the function $h$ satisfies the identities (5.3) with some $\gamma>0$. Since $G A_{u^{* n}} h \geq 0$, from (5.3) it follows that for any $n \in \mathbb{N}$ and $x \in E$,

$$
T_{u}^{n} h(x) \geq \gamma^{n} h(x) .
$$

Moreover, using (5.1) with $y=x \star u^{\star n}$ one gets

$$
h(x) \geq Q\left(x, x \star u^{\star n}\right) h\left(x \star u^{\star n}\right)=Q\left(x, x \star u^{\star n}\right) T_{u}^{n} h(x), \quad \forall x \in E
$$

and consequently,

$$
h(x) \geq Q\left(x, x \star u^{\star n}\right) \gamma^{n} h(x), \quad \forall x \in E .
$$

Remark finally that by (5.1), every non-zero harmonic function is strictly positive everywhere on $E$, and consequently, the above inequality implies that

$$
1 \geq Q\left(x, x \star u^{\star n}\right) \gamma^{n}, \quad \forall x \in E .
$$

When combined with (3.2) the last inequality proves that

$$
\log \gamma \leq-\lim _{n} \frac{1}{n} \log Q\left(x, x \star u^{\star n}\right)=0
$$


from which it follows that

$$
\gamma \leq 1
$$

To prove that $\gamma \geq 1$, remark that by (3.2), for any $\varepsilon>0$ there exists $n_{\varepsilon}>0$ such that

$$
Q\left(x \star u^{\star n}, x\right) \geq(1-\varepsilon)^{n}, \quad \forall n \geq n_{\varepsilon} .
$$

Hence, for $n \geq n_{\varepsilon}$, using again (5.1), one gets

$$
h\left(x \star u^{\star n}\right) \geq Q\left(x \star u^{\star n}, x\right) h(x) \geq(1-\varepsilon)^{n} h(x), \quad \forall x \in E .
$$

For $n \geq n_{\varepsilon}$, the function $T_{u^{\star n} h}-(1-\varepsilon)^{n} h$ is therefore positive and super harmonic. Hence, using the same arguments as in the proof of Lemma 5.2 we obtain

$$
T_{u^{\star n}} h-(1-\varepsilon)^{n} h=\gamma_{n}^{\prime} h+G \varphi_{n}^{\prime}
$$

with some $\gamma_{n}^{\prime} \geq 0$ and some potential function $G \varphi_{n}^{\prime}$. By uniqueness of the Riesz decomposition, comparison of the above relation with (5.3) proves that for $n \geq n_{\varepsilon}$,

$$
\gamma^{n}=(1-\varepsilon)^{n}+\gamma_{n}^{\prime} \geq(1-\varepsilon)^{n}
$$

and consequently, $\gamma \geq 1-\varepsilon$. Since $\varepsilon>0$ is arbitrary, from this it follows that $\gamma \geq 1$, and hence using (5.6) we conclude that

$$
\gamma=1
$$

For a minimal harmonic function $h$, Theorem 1 is therefore proved.

To extend this result for an arbitrary harmonic function $h$, we use the Poisson-Martin representation theorem : for any harmonic function $h$ there exist a positive measure $\nu_{h}$ on the minimal Martin boundary $\partial_{m} E$ relative to the Markov chain $(X(t))$ such that

$$
h(x)=\int_{\partial_{m} E} h_{\theta}(x) d \nu_{h}(\theta), \quad x \in E,
$$

where $h_{\theta}$ is a minimal harmonic function corresponding to the point $\theta \in \partial_{m} E$. Since for every $\theta \in \partial_{m} E$, the following relations

$$
h_{\theta}(x \star u)=T_{u} h_{\theta}=h_{\theta}+G A_{u} h_{\theta}, \quad u \in E,
$$

are already proved, from (5.7) by the Fubini-Tonelli theorem, one gets (3.3).

\section{Proof of Theorem 2}

6.1. Preliminary results. Consider a sequence of functions $\left(f_{n}\right)$ defined by

$$
f_{n}(x)=\mathbb{P}_{x}\left(\tau_{\vartheta}>n\right) / \mathbb{P}_{e}\left(\tau_{\vartheta}>n\right), \quad x \in E .
$$

We begin the proof of Theorem 2 with the following lemma.

Lemma 6.1. Suppose that a Markov chain $(X(t))$ satisfies the conditions (AO), (B1) and (B2). Then the following assertions hold:

1) The sequence of functions $\left(f_{n}\right)$ is relatively compact with respect to the topology of point-wise convergence.

2) For any convergent subsequence $f_{n_{k}}$, the function

$$
f(x)=\lim _{k \rightarrow \infty} f_{n_{k}}(x), \quad x \in E,
$$

is super harmonic for the Markov chain $(X(t))$ and satisfies the inequalities

$$
\mathbb{E}_{x}\left(f\left(H_{1}\right), \mathcal{T}_{\vartheta}>1\right) \leq f(x)-1, \quad \forall x \in E .
$$

3) The function $V(x)=\mathbb{E}_{x}\left(\mathcal{T}_{\vartheta}\right)$ is finite everywhere on $E$ and satisfies the inequality

$$
V(x) \leq \liminf _{n \rightarrow \infty} f_{n}(x), \quad \forall x \in E .
$$

4) The function $P V$ is finite everywhere on $E$ and satisfies the inequality $P V(e) \leq 1$. 
Proof. Indeed, since for any $n \geq 0$ and $x \in E$,

$$
f_{n}(x)=\mathbb{P}_{x}\left(\tau_{\vartheta}>n\right) / \mathbb{P}_{e}\left(\tau_{\vartheta}>n\right) \geq \mathbb{P}_{x}\left(\tau_{\vartheta}>n+1\right) / \mathbb{P}_{e}\left(\tau_{\vartheta}>n\right)=P f_{n}(x)
$$

the functions $f_{n}$ are super harmonic for $(X(t))$. Using (5.1) we conclude therefore that for any $x \in E$,

$$
Q(x, e)=Q(x, e) f_{n}(e) \leq f_{n}(x) \leq \frac{1}{Q(e, x)} f_{n}(e)=\frac{1}{Q(e, x)}, \quad \forall n \geq 0,
$$

and consequently, the sequence of functions $\left(f_{n}\right)$ is relatively compact with respect to the topology of point-wise convergence.

Consider now a convergent subsequence $\left(f_{n_{k}}\right)$. Then the function $f=\lim _{k \rightarrow \infty} f_{n_{k}}$ is super harmonic as a limit of super harmonic functions. Before proving (6.2) in our general setting let us consider the case when (B2) holds with the equality. Recall that by Proposition 3.2, in this particular case, in distribution

$$
H_{1}= \begin{cases}X\left(t_{1}\right) & \text { if } t_{1}<\infty \\ \vartheta, & \text { otherwise }\end{cases}
$$

where $t_{1}=\eta_{x}=\inf \{n>0: X(n) \notin E \star X(0)\}$, and moreover for any $x \in E$ and $n \geq 0$,

$$
P_{x}\left(t_{1}>n\right)=\mathbb{P}_{0}\left(\tau_{\vartheta}>n\right) .
$$

Hence, in the case when (B2) holds with the equality, (6.2) follows by Fatou lemma from the inequalities

$$
\begin{aligned}
\mathbb{P}_{x}\left(\tau_{\vartheta}>n\right) & =\mathbb{P}_{0}\left(\tau_{\vartheta}>n\right)+\sum_{y \in E \backslash(E \star x)} \sum_{k=1}^{n} \mathbb{P}_{x}\left(X\left(t_{1}\right)=y, t_{1}=k\right) \mathbb{P}_{y}\left(\tau_{\vartheta}>n-k\right) \\
& \geq \mathbb{P}_{0}\left(\tau_{\vartheta}>n\right)+\sum_{y \in E \backslash(E \star x)} \sum_{k=1}^{n} \mathbb{P}_{x}\left(X\left(t_{1}\right)=y, t_{1}=k\right) \mathbb{P}_{y}\left(\tau_{\vartheta}>n\right)
\end{aligned}
$$

To prove (6.2) in our general setting recall that, according to the definition of $A_{x}, T_{x} P=$ $P T_{x}+A_{x}$ and consequently,

$$
T_{x} P^{k+1}=P T_{x} P^{k}+A_{x} P^{k}, \quad \forall k \in \mathbb{N} .
$$

By iterating the last relation on gets

$$
T_{x} P^{n}=P^{n} T_{x}+\sum_{k=1}^{n} P^{k-1} A_{x} P^{n-k}, \quad \forall n \geq 1,
$$

from which it follows that

$$
T_{x} P^{n} 1=P^{n} T_{x} 1+\sum_{k=1}^{n} P^{k-1} A_{x} P^{n-k} 1=P^{n} 1+\sum_{k=1}^{n} P^{k-1} A_{x} P^{n-k} 1, \quad \forall n \geq 1 .
$$

Since for any $y \in E$,

$$
P^{n-k} 1(y)=\mathbb{P}_{y}\left(\tau_{\vartheta}>n-k\right) \geq \mathbb{P}_{y}\left(\tau_{\vartheta}>n\right)=P^{n} 1(y)
$$

and $f_{n}(y)=P^{n} 1(y) / P^{n} 1(e)$, we conclude that

$$
T_{x} f_{n} \geq \sum_{k=1}^{n} P^{k-1} A_{x} f_{n}+f_{n}, \quad \forall n \geq 1, x \in E,
$$

and in particular, since $f_{n}(e)=1$,

$$
f_{n}(x)=T_{x} f_{n}(e) \geq \sum_{k=1}^{n} P^{k-1} A_{x} f_{n}(e)+1, \quad \forall n \geq 1, x \in E .
$$


Letting therefore $n=n_{k} \rightarrow \infty$ and using Fatou lemma, one gets

$$
f(x) \geq \sum_{k=1}^{\infty} P^{k-1} A_{x} f(e)+1=G A_{x} f(e)+1, \quad \forall x \in E .
$$

Since, by definition of the ladder process $\left(H_{n}\right)$,

$$
\mathbb{E}_{x}\left(f\left(H_{1}\right), \mathcal{T}_{\vartheta}>1\right)=G A_{x} f(e)
$$

(6.4) proves (6.2). The first and the second assertions of our lemma are proved.

To prove the last assertion, we use again (6.3). By Fatou lemma, from (6.3) it follows that for $f=\liminf _{n} f_{n}(x)$ the inequalities (6.4) and (6.2) also hold. The iterates of (6.2) show that

$$
0 \leq \mathbb{E}_{x}\left(f\left(H_{n}\right), \mathcal{T}_{\vartheta}>n\right) \leq f(x)-\sum_{k=0}^{n-1} \mathbb{P}_{x}\left(\mathcal{T}_{\vartheta}>k\right), \quad \forall n \geq 0, x \in E .
$$

Letting therefore $n \rightarrow \infty$ one gets

$$
V(x)=\mathbb{E}_{x}\left(\mathcal{T}_{\vartheta}\right) \leq f(x), \quad \forall x \in E .
$$

The third assertion of our lemma is therefore proved. Moreover, the function $f$ being superhamonic, the fourth assertion of Lemma 6.1 follows from the above inequality in a straightforward way:

$$
P V(x) \leq P f(x) \leq f(x)<\infty, \quad \forall x \in E
$$

and in particular,

$$
P V(e) \leq f(e)=1
$$

As a straightforward consequence of the last assertion of Lemma 6.1 we obtain

Lemma 6.2. Under the hypotheses (A0), (B1) and (B2), for any $x \in E$, the functions $T_{x} P V, P T_{x} V$ and $A_{x} V$ are finite everywhere on $E$

Proof. Indeed, by Lemma 6.1 the function $P V$ is finite on $E$ and hence, for any $x \in E$, the function $T_{x} P V$ is also finite. Since according to the definition of the matrix $A_{x}$,

$$
T_{x} P V=P T_{x} V+A_{x} V
$$

with non negative $A_{x} V$ and $P T_{x} V$, we conclude therefore that the functions $A_{x} V$ and $P T_{x} V$ are also finite.

The following statement is the main technical point of our proof.

Proposition 6.1. Under the hypotheses (A0), (B1) and (B2), the function $V$ satisfies (3.12).

The proof of this statement is given in Section 8 . Using this result we obtain

Lemma 6.3. Under the hypotheses (A0), (B1) and (B2), the function $V$ is super harmonic for the Markov chain $(X(t)), V(e)=1$ and the function $(\mathbb{I}-P) V$ is constant in E.

Proof. Indeed, recall that by Lemma 6.1 and Lemma 6.1 the functions $P V, T_{u} P V, A_{u} V$ and $P T_{u} V$ are finite on $E$. Since by (3.12), $T_{u} V \geq G A_{u} V$, we conclude therefore that the function $G A_{u} V$ is also finite and moreover potential for $(X(t))$ with

$$
(\mathbb{I}-P) G A_{u} V=A_{u} V \text {. }
$$

The function $P G A_{u} V \leq G A_{u} V$ is therefore also finite. Hence, from (3.12) it follows that

$$
\begin{aligned}
(\mathbb{I}-P) T_{u} V & =(\mathbb{I}-P) V+(\mathbb{I}-P) G A_{u} V=(\mathbb{I}-P) V+A_{u} V \\
& =(\mathbb{I}-P) V+T_{u} P V-P T_{u} V,
\end{aligned}
$$


and consequently,

$$
T_{u}(\mathbb{I}-P) V=(\mathbb{I}-P) V, \quad \forall u \in E .
$$

The last relation shows that the function $(\mathbb{I}-P) V$ is constant on $E$. Remark now that $A_{e}=0$, and

$$
\mathbb{E}_{e}\left(H_{1}=y\right)=p_{H}(e, y)=G A_{e} 1_{\{y\}}(u)=0 \quad \forall y \in E .
$$

Hence, $\mathbb{P}_{e}\left(H_{1}=\vartheta\right)=p_{H}(e, \vartheta)=1$ and consequently, $V(e)=\mathbb{E}_{e}\left(\mathcal{T}_{\vartheta}\right)=1$. Now, to prove that the function $V$ is super harmonic for $(X(t))$ it is sufficient to notice that by Lemma 6.1

$$
P V(e) \leq 1=V(e)
$$

Lemma 6.3 is therefore proved.

Lemma 6.4. Suppose that the conditions (A0), (B1) and (B2) are satisfied and let $\mathbb{E} .\left(\tau_{\vartheta}\right)=+\infty$. Then the function $V$ is harmonic for $(X(t))$ and

$$
\lim _{n \rightarrow \infty} f_{n}(x)=V(x), \quad \forall x \in E .
$$

Proof. Indeed, recall that by Lemma 6.1, the function $V$ is super harmonic for $(X(t))$. Hence, by the Riesz decomposition theorem,

$$
V=h+G \varphi
$$

with a harmonic for $(X(t))$ function $h=\lim _{n} P^{n} V$ and $\varphi=(\mathbb{I}-P) V$. Recall moreover that by Lemma 6.3 the function $\varphi=(\mathbb{I}-P) V$ is constant on $E$. Hence, by Fubini-Tonelli theorem,

$$
G \varphi(x)=\varphi(e) G 1(x)=\varphi(e) \mathbb{E}_{x}\left(\tau_{\vartheta}\right)
$$

and consequently,

$$
V(x)=h+\varphi(e) \mathbb{E}_{x}\left(\tau_{\vartheta}\right) \geq \varphi(e) \mathbb{E}_{x}\left(\tau_{\vartheta}\right) .
$$

The last relations prove that $\varphi=\varphi(e) 1=0$ and $V=h$ whenever $\mathbb{E} .\left(\tau_{\vartheta}\right)=+\infty$.

To prove (6.5) recall that by Lemma 6.1, if a subsequence $\left(f_{n_{k}}\right)$ converges pointwisely on $E$, then the limit $f=\lim _{k \rightarrow \infty} f_{n_{k}}$ is a super harmonic function for $(X(t))$ and satisfies the inequality $V \leq f$. The function $V$ being harmonic, we conclude therefore that the function $f-V$ is super harmonic with $(f-V)(e)=0$. The Markov chain $(X(t))$ being irreducible, by the minimum principle, from this it follows that $f-V=0$ and consequently,

$$
\lim _{k \rightarrow \infty} f_{n_{k}}=V
$$

for any convergent subsequence $\left(f_{n_{k}}\right)$. The sequence $\left(f_{n}\right)$ being relatively compact with respect to the topology of point wise convergence, the last equality proves [6.5]).

Lemma 6.5. If the conditions (A0), (B1) and (B2) are satisfied and $\mathbb{E} .\left(\tau_{\vartheta}\right)<\infty$, then the function $g(x)=\mathbb{E}_{x}\left(\tau_{\vartheta}\right)$ is potential with $(\mathbb{I}-P) g=1$ and

$$
g(u) \geq g(e)+\mathbb{E}_{u}\left(g\left(H_{1}\right), \mathcal{T}_{\vartheta}>1\right), \quad \forall u \in E .
$$

Proof. Indeed, if $\mathbb{E} .\left(\tau_{\vartheta}\right)<\infty$ then by Fubini-Tonelli theorem, $G 1=\mathbb{E}$. $\left(\tau_{\vartheta}\right)$ and consequently, the function $g(x)=\mathbb{E}_{x}\left(\tau_{\vartheta}\right)$ is potential with $(\mathbb{I}-P) g=1$. Furthermore, for any $u \in E$, using the identity $T_{u} P=P T_{u}+A_{u}$ one gets

$$
P T_{u} g+A_{u} g=T_{u} P g=T_{u}(g-1)=T_{u} g-1
$$

or equivalently, since the both terms of the left hand side of the above equality are positive and the right hand side is finite,

$$
(\mathbb{I}-P) T_{u} g=1+A_{u} g \geq 0 .
$$

For any $u \in E$, the function $T_{u} g$ is therefore super harmonic for $(X(t))$ and by Riesz decomposition theorem,

$$
T_{u} g(x)=\tilde{g}(x)+G\left(1+A_{u} g\right)(x), \quad \forall x \in E,
$$


with $\tilde{g}=\lim _{n} P^{n} T_{u} g \geq 0$. Using the last relation with $x=e$ we obtain

$$
g(u)=T_{u} g(e)=\tilde{g}(e)+G 1(e)+G A_{u} g(e) \geq g(e)+G A_{u} g(e) .
$$

According to the definition of the Markov process $\left(H_{n}\right)$, the last relation proves (6.6).

Now we are ready to complete the proof of Theorem 2

6.2. Proof of Theorem 2, Suppose that the conditions (A0), (B1) and (B2) are satisfied. Then by Lemma 6.1 the function $V=\mathbb{E} .\left(\mathcal{T}_{\vartheta}\right)=G_{H} 1$ is finite everywhere on $E$ and by Lemma 6.3. $V(e)=1$. This proves that the Markov chain $\left(H_{n}\right)$ is transient.

Furthermore, $(3.12)$ is proved by Proposition 6.1. If moreover, $\mathbb{E} .\left(\tau_{\vartheta}\right)=\infty$, then by by Lemma 6.4 the function $V$ is harmonic for the Markov chain $(X(t))$ and (3.13) holds. The first tree assertions of Theorem 2 are therefore proved.

Suppose now that $\mathbb{E} .\left(\tau_{\vartheta}\right)<\infty$. Then by Lemma 6.3 , the function $V$ is super harmonic for the Markov chain $(X(t))$ and the function $(\mathbb{I}-P) V$ is constant on $E$. Moreover, by Lemma 6.5, the function $g=G 1=\mathbb{E} .\left(\tau_{\vartheta}\right)$ satisfies the relations (6.6) and consequently, it is super harmonic for the ladder height process $\left(H_{n}\right)$ with $\left(\mathbb{I}-P_{H}\right) g \geq g(e)$. Hence, by the Riesz decomposition theorem, for any $x \in E$,

$$
g(x) \geq g(e) G_{H} 1(x)+\lim _{n} P_{H}^{n} g(x) \geq g(e) G_{H} 1(x)=g(e) V(x)
$$

The function $g$ being potential for $(X(t))$, from the last inequality it follows that the super harmonic function $V$ is also potential for $(X(t))$. Since the function $(\mathbb{I}-P) V$ is constant on $E$, this proves that $V=c G 1$ with some $c>0$, and consequently, the functions $V$ and $g$ are proportional to each other. Finally, to get the equality $V=g / g(e)$ it is sufficient to notice that $V(e)=1$.

\section{Proof of Theorem 3}

Suppose that $h: E \rightarrow \mathbb{R}_{+}$is a harmonic function of $(X(t))$. Put $h(\vartheta)=0$. Then using the definition of the ladder height process $\left(H_{n}\right)$ and (3.3) with $u=x$ and $y=e$ one gets

$$
h(x)=h(e)+\mathbb{E}_{x}\left(h\left(H_{1}\right)\right), \quad x \in E .
$$

This relation shows that any harmonic function $h$ of $(X(t))$ is super harmonic for $\left(H_{n}\right)$ with $\left(\mathbb{I}-P_{H}\right) h=h(e)$. By Riesz decomposition theorem, from this it follows that

$$
h=h(e) G_{H} 1+\tilde{h}
$$

where the function $\tilde{h}=\lim _{n} P_{H}^{n} h$ is harmonic for $\left(H_{n}\right)$. The last relation proves 3.15) because by Fubini-Tonelli theorem, $G_{H} 1(x)=\mathbb{E}_{x}\left(\mathcal{T}_{\vartheta}\right)=V(x)$ The first assertion of Theorem 3 is therefore proved.

Suppose now that $\mathbb{E} .\left(\tau_{\vartheta}\right)=+\infty$. Then by Theorem 2 the function $V$ is harmonic for $(X(t))$. By (3.15), for any harmonic function $h$, the function $\tilde{h}=h-h(e) V$ is therefore also harmonic for $(X(t))$ with $\tilde{h}(e)=0$ because $V(e)=1$. By the minimum principle, this proves that $\tilde{h}=0$ and consequently, the function $h$ is proportional to $V$.

If $\mathbb{E} .\left(\tau_{\vartheta}\right)<+\infty$, then by Theorem 2 , the function $V$ is potential for $(X(t))$ and hence, for any harmonic function $h$, the function $\tilde{h}=h-h(e) V$ is non zero.

\section{Proof of Proposition 6.1}

Before proving this proposition in our general setting let us consider the main ideas of the proof in the case when (B2) holds with the equality. From now on throughout this section the assumption (A0) and (B1) are assumed satisfied. 
If (B2) holds with the equality, the ladder height process $\left(H_{n}\right)$ is given by (3.9), the equation (3.12) is equivalent to (3.5) for $h=V$, and the transition probabilities of $\left(H_{n}\right)$ satisfy the following relations

$$
p_{H}(x, y)=G A_{x} 1_{\{y\}}(e)= \begin{cases}\sum_{z \in E} G(e, z) p(z \star x, y) & \text { if } y \in E \backslash(E \star x), \\ 0 & \text { otherwise. }\end{cases}
$$

Hence, for any $u, x, y \in E$,

$$
p_{H}(x \star u, y \star u)=p_{H}(x, y),
$$

and consequently, letting for $u \in E$,

$$
\nu_{u}=\inf \left\{n \geq 0: H_{n} \notin E \star u\right\}
$$

and using the inequality $\mathcal{T}_{\vartheta} \geq \nu_{u}$, one gets

$$
\begin{aligned}
\mathbb{P}_{x \star u}\left(\mathcal{T}_{\vartheta}>n\right)=\mathbb{P}_{x \star u}\left(\nu_{u}>n\right) & \\
& +\sum_{k=1}^{n} \sum_{z \in E \backslash(E \star u)} \mathbb{P}_{x \star u}\left(H_{k}=z, \nu_{u}=k\right) \mathbb{P}_{z}\left(\mathcal{T}_{\vartheta}>n-k\right)
\end{aligned}
$$

with

$$
\mathbb{P}_{x \star u}\left(\nu_{u}>n\right)=\mathbb{P}_{x}\left(\mathcal{T}_{\varphi}>n\right) .
$$

From the two last relations using the identities

$$
V(x)=\mathbb{E}_{x}\left(\mathcal{T}_{\vartheta}\right)=\sum_{n=0}^{\infty} \mathbb{P}_{x}\left(\mathcal{T}_{\vartheta}>n\right)
$$

it follows that

$$
V(x \star u)=V(x)+\mathbb{E}_{x \star u}\left(V\left(H_{\nu_{u}}\right), \nu_{u}<\mathcal{T}_{\vartheta} \leq+\infty\right)
$$

and using moreover (3.9) we conclude that

$$
V(x \star u)=V(x)+\mathbb{E}_{x \star u}\left(V\left(X\left(t_{\nu_{u}}\right)\right), \nu_{u}<\mathcal{T}_{\vartheta} \leq+\infty\right) .
$$

To complete the proof of (3.5) for $V=h$ it is therefore sufficient to show that

$$
\mathbb{E}_{x \star u}\left(V\left(X\left(t_{\nu_{u}}\right)\right), \nu_{u}<\mathcal{T}_{\vartheta} \leq+\infty\right)=\mathbb{E}_{x \star u}\left(V\left(X\left(\eta_{u}\right)\right), \eta_{u}<\tau_{\vartheta} \leq+\infty\right) .
$$

The last relation follows from (3.9) because the event $\left\{\nu_{u}<\mathcal{T}_{\vartheta} \leq+\infty\right\}$ is equivalent to the event $\left\{\eta_{u}<\tau_{\vartheta} \leq+\infty\right\}$, and on the event $\left\{\eta_{u}<\tau_{\vartheta} \leq+\infty\right\}$, the stopping times $t_{1}, \ldots, t_{\nu_{u}}$ are finite with $t_{\nu_{u}}=\eta_{u}$. In the case when (B2) holds with the equality, Proposition 6.1 is therefore proved.

To prove (3.12) in our general setting we construct two Markov chains $\left(H_{n}\right)$ and $\left(H_{n}^{u}\right)$ on the same probability space, with the same transition probabilities (3.6) and (3.7), and such that $H_{n} \star u=H_{n}^{u}$ for any $n \leq \mathcal{T}_{\vartheta}=\inf \left\{k>0: H_{k}=\vartheta\right\}$. For this we need the following lemmas.

Lemma 8.1. Under the hypotheses (A0), (B1) and (B2), for any $x, u \in E$,

$$
A_{x \star u}=A_{x} T_{u}+T_{x} A_{u}
$$

Proof. Indeed, according to the definition of the matrices $A_{x}, x \in E$,

$$
\begin{aligned}
A_{x} T_{u}+T_{x} A_{u} & =T_{x} P T_{u}-P T_{x} T_{u}+T_{x} T_{u} P-T_{x} P T_{u}=T_{x} T_{u} P-P T_{x} T_{u} \\
& =T_{x \star u} P-P T_{x \star u}=A_{x \star u}
\end{aligned}
$$

Using this lemma we get

Lemma 8.2. Under the hypotheses (A0), (B1) and (B2), transition probabilities of the ladder process $\left(H_{n}\right)$ satisfy the following relations

$$
p_{H}(x \star u, y \star u)=p_{H}(x, y)+G T_{x} A_{u} 1_{\{y \star u\}}(e) \geq p_{H}(x, y), \quad \forall x, y, u \in E,
$$


Proof. By Lemma 8.1 from the definition of the transition probabilities $p_{H}(x, y), x, y \in E$, it follows that for any $x, y \in E$,

$$
p_{H}(x \star u, y \star u)=G A_{x \star u} 1_{\{y \star u\}}(e)=G A_{x} T_{u} 1_{\{y \star u\}}(e)+G T_{x} A_{u} 1_{\{y \star u\}}(e)
$$

Since $T_{u} 1_{\{y \star u\}}=1_{\{y\}}, G A_{x} 1_{\{y\}}(e)=p_{H}(x, y)$ and $G T_{x} A_{u} 1_{\{y \star u\}}(e) \geq 0$, the last relation proves (8.1).

Now we are ready to introduce the process $\left(H_{n}^{u}, H_{n}\right)$. We define $\left(H_{n}^{u}, H_{n}\right)$ as a Markov chain on

$$
\tilde{E}=\{(y \star u, y), \quad y \in E\} \cup\{(y, \vartheta), y \in E\} \cup\{(\vartheta, \vartheta)\}
$$

with absorbing state $(\vartheta, \vartheta)$ and transition probabilities

$$
\mathbb{P}_{\left(y^{u}, y\right)}\left(\left(H_{1}^{u}, H_{1}\right)=\left(z^{u}, z\right)\right)=\tilde{p}_{H}\left(\left(y^{u}, y\right),\left(z^{u}, z\right)\right), \quad\left(y^{u}, u\right),\left(z^{u}, z\right) \in \tilde{E}
$$

such that for $y, z \in E$,

$$
\begin{gathered}
\tilde{p}_{H}((y \star u, y),(z \star u, z))=p_{H}(y, z), \\
\tilde{p}_{H}((y \star u, y),(z \star u, \vartheta))=p_{H}(y \star u, z \star u)-p_{H}(y, z)=G T_{y} A_{u} 1_{\{z \star u\}}(e) \\
\tilde{p}_{H}((y \star u, y),(z, \vartheta))=p_{H}(y \star u, z) \text { if } z \in E \backslash E \star u, \\
\tilde{p}_{H}((y \star u, y),(\vartheta, \vartheta))=1-\sum_{z \in E} p_{H}(y \star u, z)
\end{gathered}
$$

and

$$
\tilde{p}_{H}\left((y, \vartheta),\left(z, z^{\prime}\right)\right)= \begin{cases}p_{H}(y, z) & \text { if } z^{\prime}=\vartheta \text { and } z \in E, \\ p_{H}(y, \vartheta) & \text { if } z=z^{\prime}=\vartheta, \\ 0 & \text { otherwise }\end{cases}
$$

By Lemma 8.2, under the hypotheses (A0), (B1) and (B2), the coefficients of the matrix $\left(\tilde{p}_{H}\left(v, v^{\prime}\right) ; v, v^{\prime} \in \tilde{E}\right)$ are non negative and for any $v \in \tilde{E}$,

$$
\sum_{v^{\prime} \in \tilde{E}} \tilde{p}_{H}\left(v, v^{\prime}\right)=1
$$

The Markov chain $\left(H_{n}^{u}, H_{n}\right)$ is therefore well defined. For $\left(y^{u}, y\right) \in \tilde{E}$, we denote respectively by $\mathbb{P}_{y^{u}, y}$ and $\mathbb{E}_{y^{u}, y}$ the probability measure and the corresponding expectation given that $H_{0}^{u}=y^{u}$ and $H_{0}=y$.

Remark that according to the above definition, $\left(H_{n}^{u}\right)$ and $\left(H_{n}\right)$ are two Markov chains with the same transition probabilities defined by (3.6) and (3.7). For any $\left(y^{u}, y\right) \in \tilde{E}$, and any integrable and $\sigma\left(\left(H_{n}\right)\right)$-measurable random variable $W$, we have therefore

$$
\mathbb{E}_{y^{u}, y}(W)=\mathbb{E}_{y}(W)
$$

and similarly, for any integrable and $\sigma\left(\left(H_{n}^{u}\right)\right)$ - measurable random variable $W^{u}$,

$$
E_{y^{u}, y}\left(W^{u}\right)=\mathbb{E}_{y^{u}}\left(W^{u}\right) .
$$

In particular, for $\mathcal{T}_{\vartheta}=\inf \left\{k>0: H_{k}=\vartheta\right\}$ and $\mathcal{T}_{\vartheta}^{u}=\inf \left\{n>0: H_{n}^{u}=\vartheta\right\}$,

$$
V(x \star u)=\mathbb{E}_{x \star u, x}\left(\mathcal{T}_{\vartheta}^{u}\right), \quad V(x)=\mathbb{E}_{x \star u, x}\left(\mathcal{T}_{\vartheta}\right) \quad \text { and } \quad V(z)=\mathbb{E}_{z, \vartheta}\left(\mathcal{T}_{\vartheta}^{u}\right)
$$

Remark moreover, that for any $x \in E, \mathbb{P}_{x \star u, x}$-almost surely,

$$
H_{n}^{u}=H_{n} \star u \in E \star u \subset E, \quad \forall n<\mathcal{T}_{\vartheta} .
$$

The stopping times $\mathcal{T}_{\vartheta}$ and $\mathcal{T}_{\vartheta}^{u}$ satisfy therefore the inequality

$$
\mathcal{T}_{\vartheta}^{u} \geq \mathcal{T}_{\vartheta},
$$

and using the same arguments as in the previous particular case, we obtain

$$
\begin{aligned}
\mathbb{P}_{x \star u, x}\left(\mathcal{T}_{\vartheta}^{u}>n\right)=\mathbb{P}_{x \star u, x}\left(\mathcal{T}_{\vartheta}>n\right) & \\
& +\sum_{k=1}^{n} \sum_{z \in E} \mathbb{P}_{x \star u, x}\left(H_{k}^{u}=z, \mathcal{T}_{\vartheta}=k\right) \mathbb{P}_{z, \vartheta}\left(\mathcal{T}_{\vartheta}^{u}>n-k\right) .
\end{aligned}
$$


From the last relation using (8.2) one gets

$$
V(x \star u)=V(x)+\mathbb{E}_{x \star u, x}\left(V\left(H_{\mathcal{T}_{\vartheta}}^{u}\right), \mathcal{T}_{\vartheta}<\mathcal{T}_{\vartheta}^{u} \leq+\infty\right) .
$$

Now, to complete the proof of (3.12) it is sufficient to show that

$$
\mathbb{E}_{x \star u, x}\left(V\left(H_{\mathcal{T}_{\vartheta}}^{u}\right), \mathcal{T}_{\vartheta}<\mathcal{T}_{\vartheta}^{u} \leq+\infty\right)=G A_{u} V(x), \quad \forall x, u \in E .
$$

To prove this equality we use the following two lemmas.

Lemma 8.3. Under the hypotheses (A0), (B1) and (B2), for any $u \in E$, the function

$$
F_{u}(x)=E_{x \star u, x}\left(V\left(H_{\mathcal{T}_{\vartheta}}^{u}\right), \mathcal{T}_{\vartheta}<\mathcal{T}_{\vartheta}^{u} \leq+\infty\right)
$$

is potential for the ladder process $\left(H_{n}\right)$ with

$$
\left(\mathbb{I}-P_{H}\right) F_{u}(x)=G T_{x} A_{u} V(e), \quad x \in E .
$$

Proof. Denote

$$
\varphi_{u}(x)=\mathbb{E}_{x \star u, x}\left(V\left(H_{1}^{u}\right), \mathcal{T}_{\vartheta}=1<\mathcal{T}_{\vartheta}^{u}\right), \quad x \in E .
$$

Then by Markov property, for any $k \geq 1, x \in E$,

$$
\begin{aligned}
& \mathbb{E}_{x \star u, x}\left(V\left(H_{k}^{u}\right), \mathcal{T}_{\vartheta}=k<\mathcal{T}_{\vartheta}^{u}\right) \\
& =\sum_{y \in E} \mathbb{P}_{x \star u, x}\left(\left(H_{k-1}^{u}, H_{k-1}\right)=(y \star u, y)\right) \mathbb{E}_{y \star u, y}\left(V\left(H_{1}^{u}\right), \mathcal{T}_{\vartheta}=1<\mathcal{T}_{\vartheta}^{u}\right) \\
& =\sum_{y \in E} \mathbb{P}_{x}\left(H_{k-1}=y\right) \varphi_{u}(y)=P_{H}^{k-1} \varphi_{u}(x)
\end{aligned}
$$

and consequently, for any $x \in E$,

$$
F_{u}(x)=\mathbb{E}_{x \star u, x}\left(V\left(H_{\mathcal{T}_{\vartheta}}^{u}\right), \mathcal{T}_{\vartheta}<\mathcal{T}_{\vartheta}^{u} \leq+\infty\right)=\sum_{k=1}^{\infty} P_{H}^{k-1} \varphi_{u}(x)=G_{H} \varphi_{u}(x) .
$$

The function $F_{u}$ is therefore potential for $\left(H_{n}\right)$ with $\left(\mathbb{I}-P_{H}\right) F_{u}=\varphi_{u}$. To complete the proof of our lemma it is now sufficient to show that

$$
\varphi_{u}(x)=G T_{x} A_{u} V(e), \quad \forall x \in E .
$$

To prove these relations we notice that, by definition of the transition probabilities $\tilde{p}((x \star$ $u, x),(y, \vartheta))$ and $p_{H}(x \star u, y)$, for any $x \in E$,

$$
\begin{aligned}
\varphi_{u}(x) & =\mathbb{E}_{x \star u, x}\left(V\left(H_{1}^{u}\right), \mathcal{T}_{\vartheta}=1<\mathcal{T}_{\vartheta}^{u}\right)=\sum_{y \in E} \tilde{p}((x \star u, x),(y, \vartheta)) V(y) \\
& =\sum_{y \in E}\left(p_{H}(x \star u, y \star u)-p_{H}(x, y)\right) V(y \star u)+\sum_{y \in E \backslash(E \star u)} p_{H}(x \star u, y) V(y) \\
& =\sum_{y \in E} p_{H}(x \star u, y) V(y)-\sum_{y \in E} p_{H}(x, y) V(y \star u) \\
& =P_{H} V(x \star u)-P_{H} T_{u} V(x)=G A_{x \star u} V(e)-G A_{x} T_{u} V(e)
\end{aligned}
$$

The last relation combined with Lemma 8.1 proves 8.5 .

Lemma 8.4. Under the hypotheses (AO), (B1) and (B2), for any $u \in E$, the function $G A_{u} V$ is potential for the ladder height process $\left(H_{n}\right)$ and for any $x \in E$,

$$
\left(\mathbb{I}-P_{H}\right) G A_{u} V(x)=G T_{x} A_{u} V(e)
$$

Proof. Recall that according to the definition of the operator $A_{x}$,

$$
T_{x} P=P T_{x}+A_{x} .
$$

From this identity it follows that for any $n \geq 1$,

$$
T_{x} P^{n}=P T_{x} P^{n-1}+A_{x} P^{n-1}=P^{n} T_{x}+\sum_{k=1}^{n} P^{k} A_{x} P^{n-k} .
$$


and consequently,

$$
P^{n} A_{u} V(x)=T_{x} P^{n} A_{u} V(e)=P^{n} T_{x} A_{u} V(e)+\sum_{k=1}^{n} P^{k-1} A_{x} P^{n-k} A_{u} V(e)
$$

Consider a sequence of operators $B_{n}$ defined on the set of non negative functions $\{\varphi$ : $\left.E \rightarrow \mathbb{R}_{+}\right\}$by

$$
B_{n} \varphi(x)=P^{n-1} A_{x} \varphi(e)=\sum_{y \in E} b_{n}(x, y) \varphi(y), \quad x \in E
$$

with

$$
b_{n}(x, y)=P^{n-1} A_{x} 1_{y}(e) \geq 0, \quad \forall x, y \in E,
$$

and let

$$
\varphi_{u, n}(x)=P^{n} T_{x} A_{u} V(e), \quad x \in E .
$$

With these notations, letting

$$
\varphi_{u}(x)=G T_{x} A_{u} V(e)
$$

we obtain

$$
\varphi_{u}(x)=\sum_{n=0}^{\infty} \varphi_{u, n}(x), \quad \forall x \in E .
$$

Moreover, by definition of the transition probabilities of the $\operatorname{process}\left(H_{n}\right)$,

$$
P_{H} \varphi(x)=\mathbb{E}_{x}\left(\varphi\left(H_{1}\right)\right)=G A_{x} \varphi(e)=\sum_{n=1}^{\infty} B_{n} \varphi(x),
$$

and using (8.7), for any $n \geq 1$, we get

$$
\begin{aligned}
P^{n} A_{u} V(x) & =\varphi_{u, n}(x)+\sum_{k=1}^{n} B_{k} P^{n-k} A_{u} V(x) \\
& =\varphi_{u, n}(x)+\sum_{k=1}^{n} \sum_{y \in E} b_{k}(x, y) P^{n-k} A_{u} V(y) .
\end{aligned}
$$

Since for $k=n, P^{n-k} A_{u} V(x)=A_{u} V(x)=\varphi_{u, 0}(x)$, the iterates of the last relations provide the following relations

$$
\begin{aligned}
P^{n} A_{u} V(x)=\varphi_{u, n}(x)+\sum_{m=1}^{n} \sum_{y_{1}, \ldots, y_{m} \in E} \sum_{\substack{k_{1}, \ldots k_{m} \geq 1: \\
k_{1}+\cdots+k_{m} \leq n}} b_{k_{1}}\left(x, y_{1}\right) \cdots b_{k_{m}}\left(y_{m-1}, y_{m}\right) \\
=\varphi_{u, n}(x)+\sum_{m=1}^{n} \sum_{\substack{k_{1}, \ldots k_{m} \geq 1: \\
k_{1}+\cdots+k_{m} \leq n}} B_{k_{1}} \ldots B_{k_{m}} \varphi_{u, n-\left(k_{1}+\cdots+k_{m}\right)}(x)
\end{aligned}
$$

When combined with $(8.8)$ and $(8.9)$ the above relations prove that for any $x \in E$,

$$
G A_{u} V(x)=\sum_{n=0}^{\infty} P^{n} A_{u} V(x)=\varphi_{u}(x)+\sum_{m=1}^{n} P_{H}^{m} \varphi_{u}(x)=G_{H} \varphi_{u}(x)
$$

and consequently, the function $G A_{u} V$ is potential for the ladder height process $\left(H_{n}\right)$ with $\left(\mathbb{I}-P_{H}\right) G A_{u} V(x)=\varphi_{u}(x)=G T_{x} A_{u} V(e)$.

Now we are ready to complete the proof of (3.12). The functions $F_{u}=T_{u} V-V$ and $G A_{u} V$ being potential for the ladder process $\left(H_{n}\right)$ with the same function $\varphi_{u}=$ $\left(\mathbb{I}-P_{H}\right)\left(T_{u} V-V\right)=\left(\mathbb{I}-P_{H}\right) G A_{u} V$ we conclude that $T_{u} V-V=G_{H} \varphi_{u}=G A_{u} V$. The last equality is equivalent to (3.12). Proposition 6.1 is therefore proved. 


\section{Proof of Proposition 4.1}

We begin the proof of Proposition 4.1 with the following preliminary results.

9.1. Preliminary results. In this subsection, the conditions ( $\mathrm{C} 0$ ') and ( $\mathrm{C}^{\prime}$ ') are assumed satisfied, but instead of the assumption (C2) we will assume that

(C2') the step generating function

$$
R(\alpha)=\sum_{x \in \mathbb{Z}^{d}} e^{\alpha \cdot x} \mu(x)
$$

is finite in a neighborhood of the set $D=\left\{\alpha \in \mathbb{R}^{d}: R(\alpha) \leq 1\right\}$ on $\mathbb{R}^{d}$ and

$$
\sum_{x \in \mathbb{Z}^{d}} x \mu(x) \neq 0
$$

Under the hypotheses (C0'), (C1') and (C2), the results of this subsection will be applied next to truncated versions of the original random walk $(X(t))$. By construction, the truncated versions of $(X(t))$ will satisfy $\left(\mathrm{C} 2{ }^{\prime}\right)$ instead of $(\mathrm{C} 2)$. As before, we denote by $G(x, y), x, y \in E$, the Green function of $(X(t))$

$$
G(x, y)=\sum_{n=0}^{\infty} \mathbb{P}_{x}(X(n)=y) .
$$

The main result of this subsection is the following statement.

Proposition 9.1. Under the hypotheses (CO') and (C1') and (C2'), for any $u, v \in \overline{\mathcal{C}} \times \overline{\mathcal{C}}$ and any sequences $\left(u_{n}\right),\left(v_{n}\right) \in E^{\mathbb{N}}$, with $\lim _{n} u_{n} / n=u$ and $\lim _{n} v_{n} / n=v$,

$$
\lim _{n \rightarrow \infty} \frac{1}{n} \log G\left(u_{n}, v_{n}\right)=-\sup _{\alpha \in D}\langle\alpha, v-u\rangle
$$

Before proving this result let us consider its following straightforward consequence.

Corollary 9.1. Under the hypotheses (CO') and (C1') and (C2'), for any $u \in E$ and uniformly on $x \in E$,

$$
\liminf _{n \rightarrow \infty} \frac{1}{n} \log Q(x, x+n u) \geq-\sup _{\alpha \in D}\langle\alpha, u\rangle
$$

and

$$
\liminf _{n \rightarrow \infty} \frac{1}{n} \log Q(x+n u, x) \geq-\sup _{\alpha \in D}\langle\alpha,-u\rangle
$$

Proof. Indeed, consider a homogeneous random walk $(S(t))$ on $\mathbb{Z}^{d}$ with transition probabilities $\mathbb{P}_{x}(S(1)=y)=\mu(y-x), \quad x, y \in \mathbb{Z}^{d}$. Our random walk $(X(t))$ is a copy of the random walk $(S(t))$ killed upon the time $\tau_{\vartheta}=\inf \{t \geq 1: S(t) \notin E\}$ :

$$
X(t)= \begin{cases}S(t), & \text { if } t<\tau_{\vartheta}, \\ \vartheta & \text { otherwise. }\end{cases}
$$

Because of the assumption (C2'), the random walk $(S(t))$ is transient on $\mathbb{Z}^{d}$ and Its Green function

satisfies the inequalities

$$
G_{S}(x, y)=\sum_{n=0}^{\infty} \mathbb{P}_{x}(S(n)=y), x, y \in \mathbb{Z}^{d}
$$

$$
G(x, y) \leq G_{S}(x, y)=G_{S}(0, y-x), \quad \forall x, y \in E .
$$

Hence, for any $x, u \in E$,

$$
Q(x, x+n u) \geq Q(0, n u)=G(0, n u) / G(n u, n u) \geq G(0, n u) / G_{S}(0,0) .
$$

When combined with (9.1), the last inequality proves (9.2). The proof of (9.3) is quite similar. 
To prove Proposition 9.1 it is sufficient to show that for any $u, v \in \overline{\mathcal{C}} \times \overline{\mathcal{C}}$, and $\left(u_{n}\right),\left(v_{n}\right) \in$ $E^{\mathbb{N}}$ with $\lim _{n} u_{n} / n=u$ and $\lim _{n} v_{n} / n=v$, the upper bound

$$
\limsup _{n \rightarrow \infty} \frac{1}{n} \log G\left(u_{n}, v_{n}\right) \leq-\sup _{\alpha \in D}\langle\alpha, v-u\rangle,
$$

and the lower bound

$$
\liminf _{n \rightarrow \infty} \frac{1}{n} \log G\left(u_{n}, v_{n}\right) \geq-\sup _{\alpha \in D}\langle\alpha, v-u\rangle .
$$

hold. The following lemma proves that these limits are well defined for all $u, v \in \overline{\mathcal{C}}$.

Lemma 9.1. Under the hypotheses $\left(C 1^{\prime}\right)$, for any $u \in \overline{\mathcal{C}}$, there is a sequence $\left(u_{n}\right) \in E^{\mathbb{N}}$ such that $u_{n} / n \rightarrow u$ as $n \rightarrow \infty$.

Proof. For $u \in \overline{\mathcal{C}}$ we denote by $[u] \in E$ a nearest to $u$ point of $E$, i.e. such that

$$
\min _{v \in E}|u-v|=|u-[u]|
$$

To prove our lemma it is sufficient to show that for any $u \in \overline{\mathcal{C}}$,

$$
\lim _{n \rightarrow \infty}\left|u-\frac{[n u]}{n}\right|=0 \text {. }
$$

Let $u \in \overline{\mathcal{C}}$. Then for any $\varepsilon>0$ there is $u_{\varepsilon} \in \mathcal{C}$ such that $\left|u-u_{\varepsilon}\right|<\varepsilon$. Moreover, the set $\mathcal{C}$ being open, there is $0<r_{\varepsilon}<\varepsilon$ for which an open ball $B\left(u_{\varepsilon}, r_{\varepsilon}\right)$ centered at $u_{\varepsilon}$ and having radius $r_{\varepsilon}$ is included to $\overline{\mathcal{C}}$. For any $n \geq 1$, we get therefore

$$
B\left(n u_{\varepsilon}, n r_{\varepsilon}\right) \subset \overline{\mathcal{C}}
$$

Since $E=\overline{\mathcal{C}} \cap \mathbb{Z}^{d}$, any open ball $B(a, r) \subset \overline{\mathcal{C}}$ with $r>d$ contains some point of the set $E$, and consequently, for $n>d / r_{\varepsilon}$,

$$
\left|u-\frac{[n u]}{n}\right| \leq\left|u-u_{\varepsilon}\right|+r_{\varepsilon} \leq\left|u-u_{\varepsilon}\right|+\varepsilon
$$

Letting therefore first $n \rightarrow \infty$ and next $\varepsilon \rightarrow 0$ one gets (9.6).

The upper bound (9.4) follows from the following statement:

Lemma 9.2. Under the hypotheses (C0'), (C1'), (C2'), for any $\alpha \in D$ and $\left(u_{n}\right),\left(v_{n}\right) \in$ $E^{\mathbb{N}}$ with $\lim _{n} u_{n} / n=u$ and $\lim _{n} v_{n} / n=v$,

$$
\limsup _{n \rightarrow \infty} \frac{1}{n} \log G\left(u_{n}, v_{n}\right) \leq-\langle\alpha, v-u\rangle .
$$

Proof. The proof of this lemma uses the method of the exponential change of measure. For $\alpha \in D$, the twisted measure $\mu_{\alpha}(x)=\exp (\langle\alpha, x\rangle) \mu(x)$ is substochastic on $\mathbb{Z}^{d}$. Consider a twisted random walk $\left(X_{\alpha}(t)\right)$ on $E$ with transition probabilities $\mathbb{P}_{x}\left(X_{\alpha}(1)=y\right)=$ $\mu_{\alpha}(y-x), x, y \in E$. Since clearly,

$$
\mathbb{P}_{x}\left(X_{\alpha}(t)=y\right)=\exp (\langle\alpha, y-x\rangle) \mathbb{P}_{x}(X(t)=y)
$$

for all $t \in \mathbb{N}$ and $x, y \in E$, the Green function $G_{\alpha}(x, y)$ of the twisted random walk $\left(X_{\alpha}(t)\right)$ satisfies the identities

$$
G_{\alpha}(x, y)=\exp (\langle\alpha, y-x\rangle) G(x, y), \quad x, y \in E,
$$

and in particular, for any $y \in E$,

$$
G_{\alpha}(y, y)=G(y, y) \leq G_{S}(0,0)
$$


where $G_{S}(x, y)$ denotes the Green function of the homogeneous random walk $(S(t))$ on $\mathbb{Z}^{d}$ with transition probabilities $\mathbb{P}_{x}(S(1)=y)=\mu(y-x), x, y \in \mathbb{Z}^{d}$. The homogeneous random walk being transient, from this it follows that

$$
\begin{aligned}
G(x, y) & \leq \exp (-\langle\alpha, y-x\rangle) G_{\alpha}(x, y) \\
& \leq \exp (-\langle\alpha, y-x\rangle) \mathbb{P}_{x}\left(X_{\alpha}(t)=y \text { for some } t \geq 0\right) G_{S}(0,0) \\
& \leq \exp (-\langle\alpha, y-x\rangle) G_{S}(0,0),
\end{aligned}
$$

and the last inequality implies (9.7).

To prove the lower bound (9.5) we need the following lemmas. The first lemma is a straightforward consequence of the communication condition $\left(\mathrm{C}^{\prime}\right)$. Recall that under the hypotheses $(\mathrm{C} 0$ '), there is a finite set

$$
\mathcal{E}_{0} \subset \operatorname{supp}(\mu)=\left\{x \in \mathbb{Z}^{d}: \mu(x)>0\right\},
$$

such that for any $x, y \in E$ there exists a sequence $x_{0}, x_{1}, \ldots, x_{m} \in E$ with $x_{0}=x, x_{m}=y$, $m \leq \kappa|y-x|$, and $x_{j}-x_{j-1} \in \mathcal{E}_{0}$ for all $j=1, \ldots, k$. Denote

$$
\delta=\min _{x \in \mathcal{E}_{0}} \mu(x)>0 .
$$

Lemma 9.3. Under the hypotheses (C0'), for any $x, y \in E$, there is $m \leq \kappa|y-x|$ such that

$$
\mathbb{P}_{x}(X(m)=y) \geq \delta^{\kappa|y-x|}
$$

Proof. Because of the assumption (C0'), for any $x, y \in E$ there is a sequence $x_{0}, x_{1}, \ldots$, $x_{m} \in E$ with $x_{0}=x, x_{m}=y$ and $m \leq \kappa|y-x|$ such that $x_{j}-x_{j-1} \in \mathcal{E}_{0}$ for all $j=1, \ldots, m$. Hence, for any $j=1, \ldots, m$, one gets

$$
\mathbb{P}_{x_{j-1}}\left(X(1)=x_{j}\right)=\tilde{\mu}\left(x_{j}-x_{j-1}\right) \geq \delta>0,
$$

and consequently,

$$
\mathbb{P}_{x}(X(m)=y) \geq \delta^{m} \geq \delta^{\kappa|y-x|}
$$

For a real number $s>0$ we denote by $[s]$ the integer part of $s$. The lower bound (9.5) will be proved in two steps. First we establish this bound for the points $u \neq v$ in the interior $\mathcal{C}$ of the cone $\overline{\mathcal{C}}$, and next we extend this bound to the whole cone $\overline{\mathcal{C}}$ by showing that the left and the right sides of (9.5) are continuous on $\overline{\mathcal{C}} \times \overline{\mathcal{C}}$. To prove the lower bound for $u, v \in \mathcal{C}$, we need

Lemma 9.4. For any $u \neq v, u, v \in \mathcal{C}$, there is a positive real number $T_{u, v}>0$ such that for any sequence $\left(u_{n}\right) \in E^{\mathbb{N}}$ with $\lim _{n} u_{n} / n=u$,

$$
\lim _{\varepsilon \rightarrow 0} \liminf _{n \rightarrow \infty} \frac{1}{n} \log \mathbb{P}_{u_{n}}\left(\left|X\left(\left[T_{u, v} n\right]\right)-v n\right| \leq \varepsilon n\right) \geq-\sup _{\alpha \in D}\langle\alpha, v-u\rangle
$$

Proof. Under the hypotheses (C0'), the function $\log R$ is strictly convex, the set $D=$ $\{\alpha: R(\alpha) \leq 1\}$ is convex and compact, the gradient $\nabla \log R(\alpha)$ exists everywhere on $\mathbb{R}^{d}$ and does not vanish on the boundary $\partial D=\left\{\alpha \in \mathbb{R}^{d}: R(\alpha)=1\right\}$, and the mapping $\alpha \rightarrow \nabla \log R(\alpha)=\nabla R(\alpha) / R(\alpha)$ determines a homeomorphism between $\partial \tilde{D}$ and the unit sphere $S^{d}$ in $\mathbb{R}^{d}$ (see [27]) For any non zero vector $u \in \mathbb{R}^{d}$ there is therefore a unique point $\alpha_{u} \in \partial D$ for which the gradient vector $\nabla R\left(\alpha_{u}\right)$ is proportional to $u$ :

$$
\frac{\nabla R\left(\alpha_{u}\right)}{\left|\nabla R\left(\alpha_{u}\right)\right|}=\frac{u}{|u|}
$$

and

$$
\sup _{\alpha \in D}\langle\alpha, u\rangle=\left\langle\alpha_{u}, u\right\rangle
$$


To prove our lemma we use large deviation estimates (see Mogulkii's theorem in the book of Dembo and Zeitouni 26]) for scaled processes

$$
Z_{n}(t)=\frac{1}{n} S([n t]), t \in \mathbb{R}_{+}
$$

where $(S(t))$ is a homogeneous random walk on $\mathbb{Z}^{d}$ with transition probabilities $\mathbb{P}_{x}(S(1)=$ $y)=\mu(y-x)$ and $[n t]$ denotes the integer part of $n t$. Recall that a function $\phi:[0, T] \rightarrow \mathbb{R}^{d}$ is absolutely continuous if there existes an integrable function $\dot{\phi}:[0, T] \rightarrow \mathbb{R}^{d}$ such that

$$
\phi(t)=\phi(0)+\int_{0}^{t} \dot{\phi}(s) d s, \quad \forall t \in[0, T] .
$$

The lower large deviation bound of Mogulkii's theorem proves that for any $T>0, \varepsilon>0$ and any absolutely continuous function $\phi:[0, T] \rightarrow \mathbb{R}^{d}$, if a sequence of points $\left(u_{n}\right) \in\left(\mathbb{Z}^{d}\right)^{\mathbb{N}}$ converges to $\phi(0)$ then

$$
\liminf _{n \rightarrow \infty} \frac{1}{n} \log \mathbb{P}_{u_{n}}\left(\sup _{t \in[0, T]}\left|Z_{n}(t)-\phi(t)\right|<\varepsilon\right) \geq-\int_{0}^{T}(\log R)^{*}(\dot{\phi}(t)) d t
$$

where $\mathbb{P}_{u_{n}}$ denotes the conditional probability given that $S(0)=u_{n}$ and $(\log R)^{*}$ denotes the convex conjugate of the function $\log R$ defined by

$$
(\log R)^{*}(w)=\sup _{\alpha \in \mathbb{R}^{d}}(\langle\alpha, w\rangle-R(\alpha)), w \in \mathbb{R}^{d} .
$$

When applied with an affine function $\phi(t)=u+w t, t \in[0, T]$, this inequality implies that

$$
\lim _{\substack{\varepsilon \rightarrow 0 \\ \varepsilon>0}} \liminf _{n \rightarrow \infty} \frac{1}{n} \log \mathbb{P}_{u_{n}}\left(\sup _{t \in[0, T]}\left|Z_{n}(t)-u-w t\right|<\varepsilon\right) \geq-T(\log R)^{*}(w) .
$$

Recall moreover that if for a given $w \in \mathbb{R}^{r}$ there is point $\alpha \in \mathbb{R}^{d}$ such that $\nabla(\log R)(\alpha)=w$ and $R(\alpha)=1$, then $(\log R)^{*}(w)=\langle\alpha, w\rangle-\log R(\alpha)=\langle\alpha, w\rangle$ and consequently, from the last inequality on gets

$$
\lim _{\substack{\varepsilon \rightarrow 0 \\ \varepsilon>0}} \liminf _{n \rightarrow \infty} \frac{1}{n} \log \mathbb{P}_{u_{n}}\left(\sup _{t \in[0, T]}\left|Z_{n}(t)-u-w t\right|<\varepsilon\right) \geq-T\langle\alpha, w\rangle .
$$

Consider now two points $u \neq v, u, v \in \mathcal{C}$ and let a sequence $\left(u_{n}\right) \in E^{\mathbb{N}}$ be such that $\lim _{n} u_{n} / n=u$. Then for the affine function $\varphi_{u, v}(t)=u+w_{v-u} t$ with $w_{v-u}=\nabla R\left(\alpha_{v-u}\right)$ and $T=T_{v-u}=|v-u| /\left|\nabla R\left(\alpha_{v-u}\right)\right|$, from the last inequality it follows that

$$
\begin{array}{r}
\lim _{\substack{\varepsilon \rightarrow 0 \\
\varepsilon>0}} \liminf _{n \rightarrow \infty} \frac{1}{n} \log \mathbb{P}_{u_{n}}\left(\sup _{t \in\left[0, T_{v-u}\right]}\left|Z_{n}(t)-\varphi_{u, v}(t)\right|<\varepsilon\right) \\
\geq-\left\langle\alpha_{v-u}, v-u\right\rangle
\end{array}
$$

because according to the definition of the mapping $u \rightarrow \alpha_{u}, T_{v-u} w_{v-u}=v-u$ and $R\left(\alpha_{v-u}\right)=1$. The set $\mathcal{C}$ being open and convex, for small $\varepsilon>0$ and large $n$, on the event

$$
\left\{\sup _{t \in\left[0, T_{v-u}\right]}\left|Z_{n}(t)-u-w_{v-u} t\right|<\varepsilon\right\},
$$

our random walk $(S(t))$ starting at $u_{n}$ does not exit from $E=\overline{\mathcal{C}} \cap \mathbb{Z}^{d}$. This proves that the left hand side of (9.11) is equal to the similar probability for the random walk $(X(t))$ starting at $X(0)=u_{n}$ and killed upon the time $\tau_{\vartheta}$ of the first exit from $E$, and consequently,

$$
\begin{array}{r}
\lim _{\substack{\varepsilon \rightarrow 0 \\
\varepsilon>0}} \liminf _{n \rightarrow \infty} \frac{1}{n} \log \mathbb{P}_{u_{n}}\left(\sup _{t \in\left[0, T_{v-u}\right]}\left|\frac{1}{n} X([n t])-u-w_{v-u} t\right|<\varepsilon, \tau_{\vartheta}>n T_{v-u}\right) \\
\geq-\left\langle\alpha_{v-u}, v-u\right\rangle .
\end{array}
$$


Remark finally that on the event

$$
\left\{\sup _{t \in\left[0, T_{v-u}\right]}\left|\frac{1}{n} X([n t])-u-w_{v-u} t\right|<\varepsilon, \tau_{\vartheta}>n T_{v-u}\right\}
$$

the inequality $\left|X\left(\left[T_{v-u} n\right]\right)-v\right| \leq \varepsilon$ holds. From (9.12) it follows therefore that

$$
\lim _{\varepsilon>0} \liminf _{n \rightarrow \infty} \frac{1}{n} \log \mathbb{P}_{u_{n}}\left(\left|X\left(\left[T_{v-u} n\right]\right)-v\right| \leq \varepsilon\right) \geq-\left\langle\alpha_{u-v}, u-v\right\rangle .
$$

When combined with (9.10) the last relation proves (9.8)

Now, we are ready to prove the lower bound (9.5) for $u \neq v, u, v \in \mathcal{C}$. This is a subject of the following lemma.

Lemma 9.5. Suppose that $u \neq v, u, v \in \mathcal{C}$ and let two sequences $\left(u_{n}\right),\left(v_{n}\right) \in E^{\mathbb{N}}$ be such that $\lim _{n} u_{n} / n=u$ and $\lim _{n} v_{n} / n=v$. Then under the hypotheses (C0'), (C1') and (C2'), 9.5 holds.

Proof. Indeed, Lemma 9.3 combined with the inequality

$$
G\left(u_{n}, v_{n}\right) \geq \sum_{z \in E:|z-n v| \leq \varepsilon n} \mathbb{P}_{u_{n}}\left(X\left(\left[T_{u, v} n\right]\right)=z\right) G\left(z, v_{n}\right)
$$

shows that for any $\varepsilon>0$,

$$
G\left(u_{n}, v_{n}\right) \geq \delta^{\kappa \varepsilon n} \mathbb{P}_{u_{n}}\left(\left|X\left(\left[T_{u, v} n\right]\right)-n v\right| \leq \varepsilon n\right)
$$

and hence, using (9.8) one gets

$$
\begin{aligned}
\liminf _{n \rightarrow \infty} \frac{1}{n} \log G\left(u_{n}, v_{n}\right) & \geq \lim _{\varepsilon \rightarrow 0} \liminf _{n \rightarrow \infty} \frac{1}{n} \log \mathbb{P}_{u_{n}}\left(\left|X\left(\left[T_{u, v} n\right]\right)-n v\right| \leq \varepsilon n\right) \\
& \geq-\sup _{\alpha \in D}\langle\alpha, v-u\rangle .
\end{aligned}
$$

Consider now for $u, v \in \overline{\mathcal{C}} \times \overline{\mathcal{C}}$

$$
J(u, v)=\liminf _{n \rightarrow \infty} \frac{1}{n} \log G\left(u_{n}, v_{n}\right)
$$

where the limit is taken over all sequences $\left(u_{n}\right),\left(v_{n}\right) \in E^{\mathbb{N}}$ with $\lim _{n} u_{n} / n=u$ and $\lim _{n} v_{n} / n=v$. Remark that by Lemma 9.1, the function $J$ is well defined everywhere on $\overline{\mathcal{C}} \times \overline{\mathcal{C}}$. To extend the lower bound (9.5) for $u, v \in \overline{\mathcal{C}} \times \overline{\mathcal{C}}$, we will use

Lemma 9.6. Under the hypotheses (C0'), (C1') and (C2'), the function $J$ is finite and continuous on $\overline{\mathcal{C}} \times \overline{\mathcal{C}}$.

Proof. Let $u, v \in \overline{\mathcal{C}} \times \overline{\mathcal{C}}$ and let two sequences $\left(u_{n}\right),\left(v_{n}\right) \in E^{\mathbb{N}}$ be such that $\lim _{n} u_{n} / n=u$ and $\lim _{n} v_{n} / n=v$. Then by Lemma9.3 for any $n \geq 0$ there ix $0 \leq m_{n} \leq \kappa\left|u_{n}-v_{n}\right|$ such that

$$
G\left(u_{n}, v_{n}\right) \geq \mathbb{P}_{u_{n}}\left(X\left(m_{n}\right)=v_{n}\right) \geq \delta^{\kappa\left|v_{n}-u_{n}\right|}
$$

and consequently,

$$
J(u, v) \geq \liminf _{n \rightarrow \infty} \frac{1}{n} \log \delta^{\kappa\left|v_{n}-u_{n}\right|}=\kappa|u-v| \log \delta
$$

Moreover, from Lemma 9.2 it follows that for any $\alpha \in D$,

$$
J(u, v) \leq-\langle\alpha, v-u\rangle \text {. }
$$

The function $J$ is therefore finite on $\overline{\mathcal{C}} \times \overline{\mathcal{C}}$. To prove its continuity, we use again Lemma 9.3. Consider $(u, v),(\hat{u}, \hat{v}) \in \overline{\mathcal{C}} \times \overline{\mathcal{C}}$ and let sequences $\left(u_{n}\right),\left(v_{n}\right),\left(\hat{u}_{n}\right),\left(\hat{v}_{n}\right) \in E^{\mathbb{N}}$ be such that

$$
\lim _{n \rightarrow \infty} u_{n} / n=u, \quad \lim _{n \rightarrow \infty} v_{n} / n=v, \quad \lim _{n \rightarrow \infty} \hat{u}_{n} / n=\hat{u}, \quad \lim _{n \rightarrow \infty} \hat{v}_{n} / n=\hat{v},
$$


and

$$
J(u, v)=\lim _{n} \frac{1}{n} \log G\left(u_{n}, v_{n}\right) .
$$

By Lemma 9.3 for any $n \geq 0$ there exist $0 \leq k_{n} \leq \kappa\left|u_{n}-\hat{u}_{n}\right|$ and $m_{n} \leq \kappa\left|v_{n}-\hat{v}_{n}\right|$ such that

$$
\mathbb{P}_{u_{n}}\left(X\left(k_{n}\right)=\hat{u}_{n}\right) \geq \delta^{\kappa\left|u_{n}-\hat{u}_{n}\right|} \text { and } \mathbb{P}_{\hat{v}_{n}}\left(X\left(m_{n}\right)=v_{n}\right) \geq \delta^{\kappa\left|v_{n}-\hat{v}_{n}\right|} .
$$

Using these inequalities together with the inequality

$$
G\left(u_{n}, v_{n}\right) \geq \mathbb{P}_{u_{n}}\left(X\left(k_{n}\right)=\hat{u}_{n}\right) G\left(\hat{u}_{n}, \hat{v}_{n}\right) \mathbb{P}_{\hat{v}_{n}}\left(X\left(m_{n}\right)=v_{n}\right)
$$

we obtain

$$
\frac{1}{n} \log G\left(u_{n}, v_{n}\right) \geq \frac{1}{n} \log G\left(\hat{u}_{n}, \hat{v}_{n}\right)+\kappa \frac{\left|u_{n}-\hat{u}_{n}\right|+\left|v_{n}-\hat{v}_{n}\right|}{n} \log \delta
$$

and letting finally $n \rightarrow \infty$ we conclude that

$$
J(u, v) \geq J(\hat{u}, \hat{v})+\kappa(|\hat{u}-u|+|\hat{v}-v|) \log \delta .
$$

Similarly

$$
J(\hat{u}, \hat{v}) \geq J(u, v)+\kappa(|\hat{u}-u|+|\hat{v}-v|) \log \delta,
$$

and consequently, for any $(u, v),(\hat{u}, \hat{v}) \in \overline{\mathcal{C}} \times \overline{\mathcal{C}}$,

$$
|J(\hat{u}, \hat{v})-J(u, v)| \leq \kappa(|\hat{u}-u|+|\hat{v}-v|)|\log \delta|
$$

The last inequality proves that the function $J$ is Lipschitz continuous on $\overline{\mathcal{C}} \times \overline{\mathcal{C}}$

Now we are ready to complete the proof of Proposition 9.1 Recall that the upper bound (9.4) is a straightforward consequence of Lemma 9.2 According to the definition of the function $J: \overline{\mathcal{C}} \times \overline{\mathcal{C}} \rightarrow \mathbb{R}$, to prove the lower bound (9.5) it is sufficient to show that

$$
J(u, v) \geq-\sup _{\alpha \in D}\langle\alpha, v-u\rangle
$$

for all $(u, v) \in \overline{\mathcal{C}} \times \overline{\mathcal{C}}$. The last inequality is already proved by Lemma 9.6 for $u \neq v, u, v \in \mathcal{C}$. To extend (9.13) for all $(u, v) \in \overline{\mathcal{C}} \times \overline{\mathcal{C}}$, it is now sufficient to notice that by Lemma 9.6. the function $J$ is continuous everywhere on $: \overline{\mathcal{C}} \times \overline{\mathcal{C}}$ and the function $w \rightarrow \sup _{\alpha \in D}\langle\alpha, w\rangle$ is continuous on $\mathbb{R}^{d}$ (see Corollary 13.2.2 in the book of Rockafellar 28] ). Proposition 9.1 is therefore proved.

9.2. Proof of Proposition 4.1. From now on the conditions (C0'), (C1') and (C2) are assumed satisfied. Since for any $x, u \in E$ and $n \geq 1$,

$$
Q(x, x+n u) \geq Q(0, n u) \text { and } Q(x+n u, x) \geq Q(n u, 0),
$$

to prove Proposition 4.1 it is sufficient to show that for any $u \in E$,

$$
\liminf _{n \rightarrow \infty} \frac{1}{n} \log Q(0, n u) \geq 0
$$

and

$$
\liminf _{n \rightarrow \infty} \frac{1}{n} \log Q(n u, 0) \geq 0
$$

To get these inequalities we introduce the following truncated and twisted processes.

Recall that by $\left(\mathrm{C}^{\prime}\right)$, there are $\kappa>0$ and a finite set $\mathcal{E}_{0} \subset \operatorname{supp}(\mu)=\left\{x \in \mathbb{Z}^{d}: \mu(x)>\right.$ $0\}$, such that for any $x \neq y, x, y \in E$, there is a sequence $x_{0}, x_{1}, \ldots, x_{n} \in E$ with $x_{0}=x$, $x_{n}=y$ and $n \leq \kappa|y-x|$ such that $x_{j}-x_{j-1} \in \mathcal{E}_{0}$ for all $j \in\{1, \ldots, n\}$. Consider a sequence of sub probability measures $\left(\mu_{k}\right)$ on $\mathbb{Z}^{d}$ such that

- $\mu_{k}(x) \neq 0$ for all $x \in \mathcal{E}_{0}$;

- for any $x \in \mathbb{Z}^{d}$, the sequence $\mu_{k}(x)$ is increasing and tends to $\mu(x)$ as $k \rightarrow \infty$; 
- for any $k \geq 1$, the set $\operatorname{supp}\left(\mu_{k}\right)=\left\{x \in \mathbb{Z}^{d}: \mu_{k}(x)>0\right\}$ is finite and

$$
\sum_{x \in \mathbb{Z}^{d}} \mu_{k}(x)<1
$$

Then for any $k \geq 1$ the function

$$
R_{k}(\alpha)=\sum_{x \in \mathbb{Z}^{d}} e^{\alpha \cdot x} \mu_{k}(x)
$$

is strictly convex and finite everywhere in $\mathbb{R}^{d}$, the set $D_{k}=\left\{\alpha \in \mathbb{R}^{d}: R_{k}(\alpha) \leq 1\right\}$ is compact and the gradient

$$
\nabla \log R_{k}(\alpha)=\frac{\nabla R_{k}(\alpha)}{\left.R_{k}(\alpha)\right)}
$$

does not vanish on the boundary $\partial D_{k}=\left\{\alpha \in \mathbb{R}^{d}: R_{k}(\alpha)=1\right\}$ (see [27]). For given $k \geq 1$ and $\alpha_{k} \in \partial D_{k}$, we put

$$
\tilde{\mu}_{k}(x)=\exp \left(\left\langle\alpha_{k}, x\right\rangle\right) \mu_{k}(x), \quad x \in \mathbb{Z}^{d},
$$

and we introduce a truncated and twisted random walk $\left(\tilde{X}_{k}(n)\right)$ on $E$ with transition probabilities

$$
\mathbb{P}_{x}\left(\tilde{X}_{k}(1)=y\right)=\tilde{\mu}_{k}(y-x)
$$

Denote by $\tilde{R}_{k}$ the generating function of $\tilde{\mu}_{k}$ :

$$
\tilde{R}_{k}(\alpha)=\sum_{x \in \mathbb{Z}^{d}} \exp (\langle\alpha, x\rangle) \tilde{\mu}_{k}(x)
$$

and let $\tilde{D}_{k}=\left\{\alpha \in \mathbb{R}^{d}: \tilde{R}_{k}(\alpha) \leq 1\right\}$. The hitting probabilities related to $\left(\tilde{X}_{k}(t)\right)$ will be denoted by

$$
\tilde{Q}_{k}(x, y)=\mathbb{P}_{x}\left(\tilde{X}_{k}(n)=y, \text { for some } n>0\right), \quad x, y \in E .
$$

Remark that the twisted measure $\tilde{\mu}_{k}$ is stochastic because for any $x \in \mathbb{Z}^{d}$,

$$
\sum_{x \in \mathbb{Z}^{d}} \tilde{\mu}_{k}(x)=\sum_{x \in \mathbb{Z}^{d}} \mu_{k}(x) \exp \left(\left\langle\alpha_{k}, y-x\right\rangle\right)=R_{k}\left(\alpha_{k}\right)=1 .
$$

Moreover, for the twisted random walk $\left(\tilde{X}_{k}(n)\right)$ the conditions $\left(\mathrm{C} 0^{\prime}\right),\left(\mathrm{C} 1^{\prime}\right)$ and $\left(\mathrm{C} 2{ }^{\prime}\right)$ of the previous subsection are clearly satisfied. Using therefore Corollary 9.1 we obtain

Corollary 9.2. Under the hypotheses (C0') and (C1') and (C2), for any $k \geq 1, u \in E$ and uniformly on $x \in E$,

$$
\liminf _{n \rightarrow \infty} \frac{1}{n} \log \tilde{Q}_{k}(0, n u) \geq-\sup _{\alpha \in \tilde{D}_{k}}\langle\alpha, u\rangle
$$

and

$$
\liminf _{n \rightarrow \infty} \frac{1}{n} \log \tilde{Q}_{k}(n u, 0) \geq-\sup _{\alpha \in \tilde{D}_{k}}\langle\alpha,-u\rangle
$$

Remark now that

$$
\begin{gathered}
\tilde{R}_{k}(\alpha)=\sum_{x \in \mathbb{Z}^{d}} \exp \left(\left\langle\alpha+\alpha_{k}, x\right\rangle\right) \mu_{k}(x)=R_{k}\left(\alpha+\alpha_{k}\right) \\
\tilde{D}_{k}=\left\{\alpha \in \mathbb{R}^{d}: R_{k}\left(\alpha+\alpha_{k}\right) \leq 1\right\}=\left\{\alpha \in \mathbb{R}^{d}: R_{k}(\alpha) \leq 1\right\}-\alpha_{k}=D_{k}-\alpha_{k} .
\end{gathered}
$$

and, by definition of the process $(\tilde{X}(t))$, for any $x, y \in E$,

$$
\tilde{Q}_{k}(x, y) \leq \exp \left(\left\langle\alpha_{k}, y-x\right\rangle\right) Q(x, y) .
$$

Hence, using (9.16) we conclude that for any $u \in E$ and $k \geq 1$,

$$
\liminf _{n \rightarrow \infty} \frac{1}{n} \log Q(0, n u) \geq-\sup _{\alpha \in D_{k}}\langle\alpha, u\rangle .
$$


Recall now that by construction, the sequence of functions $R_{k}$ is increasing and by monotone convergence theorem, $R_{k}(\alpha) \rightarrow R(\alpha)$ as $k \rightarrow \infty$ for any $\alpha \in \mathbb{R}^{d}$. The sequence of compact sets $D_{k}$ is therefore decreasing and

$$
\bigcap_{k \geq 1} D_{k}=\left\{\alpha \in \mathbb{R}^{d}: R(\alpha) \leq 1\right\}
$$

Since by (C2), $\left\{\alpha \in \mathbb{R}^{d}: R(\alpha) \leq 1\right\}=\{0\}$, letting $k \rightarrow \infty$ in (9.18) one gets (9.14). The proof of (9.15) is quite similar and uses the estimates (9.17). Proposition 4.1] is therefore proved.

\section{REFERENCES}

[1] Stanley A. Sawyer, Martin boundaries and random walks, Contemporary Mathematics, 206 (1997), no. $1,17-44$.

[2] J. L. Doob, Discrete potential theory and boundaries, J.Math. and Mech. 8 (1959), 433-458.

[3] G. A. Hunt, Markoff chains and Martin boundaries, Illinois J. Math. 4 (1960), 313-340.

[4] E.B. Dynkin, Boundary theory of Markov processes, Russian Mathematical Surveys 24 (1969), no. $7,1-42$.

[5] J. Bertoin and R. A.Doney, On Conditioning a Random Walk to Stay Nonnegative, The Annals of Probability, 4 (1994), 2152-2167.

[6] D. Denisov and V. Wachtel, Random walks in cones, Annals of Probability, 43 (2015), No. 3, $992-1044$

[7] K. Raschel and P. Tarrago, Martin boundary of random walks in convex cones, e-print arXiv: $1803.09253 \mathrm{v} 2$ [math.PR]

[8] F. Spitzer, Principles of Random Walk, 1964, Van Nostrand, Princeton.

[9] W. Feller, An introduction to probability theory and its applications, 2, 2nd ed., 1971, Wiley series in probability, New York,

[10] A.A. Mogulskii and E.A. Pecherskii, On the first exit time from a semigroup for a random walk. Theory Probab. Appl., 22(1977), 818-825.

[11] P. Greenwood and M. Shaked, Fluctuation of random walks in Rd and storage systems. Adv. Appl. Prob., 9(1977), 566-587.

[12] P. Greenwood and M. Shaked, Dual pairs of stopping times for random walk, The Annals of Probability, 6 (1978), no. 4, 644-650.

[13] I.A. Kurkova and V.A. Malyshev, Martin boundary and elliptic curves, Markov Processes Related Fields 4 (1998), 203-272.

[14] I. Kurkova, and K. Raschel, Random walks in $\mathbb{Z}_{+}^{2}$ with non-zero drift absorbed at the axes, Bull. Soc. Math. France 139 (2011) 341-387.

[15] K. Raschel, Random walks in the quarter plane, discrete harmonic functions and conformal mappings, with an appendix by Sandro Franceschi. Stochastic Processes and their Applications 124, (2014) 3147?3178. MR-3231615

[16] Irina Ignatiouk-Robert and Christophe Loree, Martin boundary of a killed random walk on a quadrant Annals of Probability, online (2010), e-print arXiv:0903.0070

[17] I. Ignatiouk-Robert. Martin boundary of a killed random walk on $\mathbb{Z}_{+}^{d}$, e-print arXiv, (2009) 1-49.

[18] Aymen Bouaziz, Sami Mustapha and Mohamed Sifi, Discrete harmonic functions on an orthant in $\mathbb{Z}^{d}$, Electron. Commun. Probab. 20 (2015), paper no. 52, 13 pp.

[19] I.M. Gessel and D. Zeilberger, Random walk in a Weyl chamber. Proc. Amer. Math. Soc. (1992) $115,27-31$.

[20] D.J. Grabiner and P. Magyar, Random walks in Weyl chambers and the decomposition of tensor powers. J. Algebraic Combin., 2 (1993), 239-260.

[21] W. Konig, and P. Schmid. Random walks conditioned to stay in Weyl chambers of type C and D, Electron. Comm. Probab. 15 (2010) 286?296.

[22] K. Raschel, Green functions and Martin compactification for killed random walks related to SU(3), Electron. Commun. Probab. 15 (2010), 176-190.

[23] K. Raschel, Green functions for killed random walks in the Weyl chamber of Sp(4), Ann. Inst. Henri Poincaré Probab. Stat. 47 (2011), 1001-1019.

[24] Jetlir Duraj, Random walks in cones: The case of nonzero drift, Stochastic Processes and their Application, 124 (2014), no. 4, 1503-1518.

[25] Wolfgang Woess, Random walks on infinite graphs and groups, Cambridge University Press, Cambridge, 2000.

[26] Amir Dembo and Ofer Zeitouni, Large deviations techniques and applications, Springer-Verlag, New York, 1998.

[27] P.L. Hennequin, Processus de Markoff en cascade, Ann. Inst. H. Poincaré 18 (1963), no. 2, 109196.

[28] R. Tyrrell Rockafellar, Convex analysis, Princeton University Press, Princeton, NJ, 1997, Reprint of the 1970 original, Princeton Paperbacks.

Université de Cergy-Pontoise, Département de mathématiques, 2, Avenue Adolphe Chauvin, 95302 Cergy-Pontoise Cedex, France

E-mail address: Irina.Ignatiouk@u-cergy.fr 